\title{
Activités
}

18-1 | 2021

Le programme de recherche cours d'action (2)

\section{Modélisation théorique de l'expérience mimétique et cours d'action : analyse de situations de formation en enseignement, santé, et sport}

Theoretical modelling of the mimetic experience and "Course of action": analysis of training situations in teaching, health and sports sectors

Serge Leblanc, Hélène Bouchot et Mélanie Secheppet

\section{OpenEdition \\ Journals}

Édition électronique

URL : http://journals.openedition.org/activites/6249

DOI : 10.4000/activites.6249

ISSN : $1765-2723$

Éditeur

ARPACT - Association Recherches et Pratiques sur les ACTivités

\section{Référence électronique}

Serge Leblanc, Hélène Bouchot et Mélanie Secheppet, « Modélisation théorique de l'expérience mimétique et cours d'action : analyse de situations de formation en enseignement, santé, et sport », Activités [En ligne], 18-1 | 2021, mis en ligne le 15 avril 2021, consulté le 17 avril 2021. URL : http:// journals.openedition.org/activites/6249; DOI : https://doi.org/10.4000/activites.6249

Ce document a été généré automatiquement le 17 avril 2021

\section{c) (†)}

Activités est mis à disposition selon les termes de la licence Creative Commons Attribution - Pas d'Utilisation Commerciale - Pas de Modification 4.0 International. 


\section{Modélisation théorique de l'expérience mimétique et cours d'action : analyse de situations de formation en enseignement, santé, et sport}

Theoretical modelling of the mimetic experience and "Course of action": analysis of training situations in teaching, health and sports sectors

Serge Leblanc, Hélène Bouchot et Mélanie Secheppet

\section{NOTE DE L'ÉDITEUR}

Article soumis le 2 mars 2020, accepté le 27 mai 2020

\section{Introduction}

1 En nous inscrivant dans le cadre du programme de recherche empirique dit du «Cours d'action » (Theureau, 2004, 2006) ou d' « anthropologie culturelle enactive » (Theureau, 2015), nous étudions les processus d'apprentissage-développement à l'œuvre dans différents dispositifs et contextes de formation professionnelle (santé, éducation, sport). Nous nous intéressons plus particulièrement à la modalité dite d'alloconfrontation ${ }^{1}$, qui consiste à confronter les formés à des films de pairs en situation de travail, de simulation ou de commentaires sur l'activité filmée. Ces dispositifs de vidéoformation contribuent à stimuler, anticiper et accompagner des apprentissages professionnels. Ces environnements sollicitent et articulent des processus mimétiques, réflexifs, projectifs et ludiques, sur la base d'une activité de comparaison entre ce que les acteurs en formation perçoivent d'une situation filmée- 
commentée ou filmée-simulée, et ce qu'ils font dans la réalité de leur travail dans des situations similaires, ou ce qu'ils feraient à la place des protagonistes filmés. Sur la base de travaux menés initialement dans des dispositifs de vidéoformation des enseignants (Gaudin, Flandin, Moussay, \& Chaliès, 2018; Leblanc, 2018 ; Leblanc, Ria, Dieumegard, Serres, \& Durand, 2008) utilisant la plateforme Néopass@ction (Ria \& Leblanc, 2011), nous avons mis en évidence que «l'efficacité d'un dispositif réside dans la forme de médiation qu'il instaure dans le rapport qu'entretiennent les enseignants formés avec leur travail, c'est-à-dire l'activité cognitive que ce dispositif tend à générer chez eux en situation de vidéoformation et les transformations réelles ou potentielles de leur activité d'enseignement " (Flandin, Leblanc, \& Muller, 2015, p. 180). La confrontation vidéoscopique d'un ou de pair(s) au travail contribue à une «mise en relation» de l'activité visionnée et de l'activité/expérience du formé. En situation de formation, cette comparaison reste le plus souvent une activité implicite, peu explorée et qui reste silencieuse. Les chercheurs ont, quant à eux, des difficultés pour documenter de manière empirique un processus complexe de mises en relation d'expériences vécues par les acteurs.

2 Cependant plusieurs recherches francophones sur la vidéoformation en éducation (Flandin et al., 2015), et des travaux menés dans le domaine de la santé (Bouchot \& Leblanc, 2019) et du sport (Secheppet, 2020), font ressortir un processus phénoménologique mimétique très présent et assez richement documenté. Or, cette faculté mimétique, contribuant à l'émergence du monde perçu, à la base de toute connaissance, est pourtant peu reconnue à la fois dans le monde de l'éducation et de la formation, et peu exploitée scientifiquement, car la conception philosophique dominante des modalités de la connaissance humaine « sous-estime l'accès non réflexif au monde que rend possible l'exemplification mimétique » (Schaeffer, 1999, p. 57). La non-prise en compte de cette dimension anthropologique fondamentale de l'activité humaine peut s'expliquer par plusieurs raisons telles que : l'attachement à une vision rationaliste de la construction des connaissances basée sur l'opposition vrai-faux, l'invisibilité des processus de simulation auxquels sont soumises les personnes, une conception sérieuse de la connaissance incompatible avec le jeu et le plaisir ou encore une vision unifiée de l'identité personnelle écartant la pluralité de nos modes d'existence.

3 À travers cette contribution, nous souhaitons rendre compte, à partir de données empiriques issues de plusieurs contextes de formation professionnelle, des différentes façons d'appréhender l'action mimétique comme «le fait "d'imiter", "d'entrer en émulation", de "faire le semblable de", ou encore de "s'approprier" (...) le fait "d'exprimer" quelque chose, de "porter quelque chose à la représentation" ou même "d'anticiper" quelque chose» (Wulf, 2007, p. 28). Nous présenterons dans un premier temps la compatibilité théorique du concept de mimesis avec les hypothèses de substance de l'enaction et de l'expérience qui organisent le programme de recherche $\mathrm{du}$ « cours d'action ». L'hypothèse de l'enaction porte sur les interactions asymétriques qu'un acteur entretient avec son environnement, dont les autres acteurs font éminemment partie. L'activité ${ }^{2}$ est donc considérée à la fois comme individuelle et sociale (Theureau, 2006). Nous nous demanderons, dans un second temps, quelle est, en situation de formation professionnelle, la nature de cette activité individuellesociale lors d'une confrontation à l'activité d'autres acteurs dans des dispositifs de vidéoformation et/ou d'observation de pairs ou d'experts. 
4 En nous inscrivant dans un situationnisme méthodologique (Theureau, 2006) qui écarte une focalisation sur les caractéristiques individuelles des acteurs (individualisme méthodologique) ou sur les caractéristiques des collectifs (collectivisme méthodologique), nous contribuerons à l'enrichissement de la connaissance de cette activité individuelle-sociale en précisant comment l'acteur en situation de formation prend en compte l'activité de l'autre ou des autres en lien avec sa propre activité. Nous développerons des hypothèses de connaissance relatives aux processus mimétique et fictionnel qui seraient à l'œuvre dans ces situations de formation. Nous mettrons à jour les conditions à réaliser pour faire s'exprimer la conscience préréflexive de l'acteur (Poizat \& San Martin, 2020 ; Theureau, 2011) lorsqu'il est confronté à l'activité de pairs (alloconfrontation) dans des situations de vidéoformation et/ou d'observation de simulation. Nous rendrons également compte, d'un point de vue théoricométhodologique, de la façon dont nous pistons des «transformations silencieuses » qui s'opèrent par la mise en relation de l'activité de pair(s) visionnée-observée avec l'activité de l'observateur-acteur sur des temps plus ou moins longs (quelques heures à plusieurs mois). Enfin, nous discuterons des apports et limites de cette contribution à la fois sur le versant théorique et sur le versant de la conception d'espaces de formation immersifs.

\section{Cadre conceptuel pour appréhender l'apprentissage-développement}

5 Chercher à comprendre l'apprentissage-développement dans des contextes où celui-ci est visé nécessite de proposer une théorie de la formation permettant de rendre compte des conditions pour accéder aux transformations inhérentes au processus d'apprentissage-développement. Cette théorie de la formation doit également permettre de rendre explicite la manière dont on croise les processus de construction de l'activité et des sujets (Barbier, 2009). Pour rendre compte de ce processus d'apprentissage-développement que nous ne réduisons ni à la « propriété » d'un sujet, ni à une "détermination » sociale, nous cherchons à appréhender, en nous appuyant sur le cadre théorique du programme de recherche du cours d'action, les "propriétés relationnelles" du tryptique acteur-environnement-autrui à travers l'analyse des transformations de l'activité. L'analyse des «cours d'action » et " cours de vie relatif à une pratique » nous permet d'appréhender la dynamique de l'activité en « pistant » les " possibles nouveaux" qui s'ouvrent dans des situations d'alloconfrontation médiées par la vidéo. Parmi ces possibles, nous nous focalisons sur ceux qui s'actualisent dans la relation entre l'activité réelle d'autrui et l'activité imaginaire de l'observateur-trice, transformant de fait les savoirs par renforcement, invalidation ou création de types ${ }^{3}$. Pour analyser les transformations de l'activité dans des situations de formation qui visent à provoquer un apprentissage-développement professionnel, nous développons deux hypothèses relatives, d'une part, aux processus d'autopoïèse et de typicalisation et, d'autre part, aux processus de mimésis et de fiction.

\subsection{Autopoïèse et typicalisation}

6 Nous concevons la construction des connaissances comme résultant d'un processus autopoïétique (Maturana \& Varela, 1994), et de typicalisation (Rosch, 1973; Theureau, 
2004). La théorie de l'autopoïèse définit l'apprentissage-adaptation comme une « dérive structurale continue» (Maturana \& Varela, 1994) dans laquelle le "couplage structural » (i.e. l'histoire continue des relations qu'un organisme vivant entretient avec son environnement) est régulé par un mécanisme d'autoréférence. Cette autoréférentialité fonctionne comme un processus de «reconnaissance» en fonction de l'identité du système, ce qui lui confère son intelligence. « Notre relation au monde se fait comme dans un miroir, qui ne nous dit ni ce qu'est le monde, ni ce qu'il n'est pas. Elle révèle seulement qu'il est possible d'avoir notre manière d'être et notre façon d'agir, et qu'ainsi notre expérience est viable » (Varela, 1989, p. 31). L'expérience de l'apprentissage-développement, conçue comme la transformation permanente de l'activité du fait de sa dynamique propre et de son ajustement à l'environnement, est guidée par ce processus d'auto-référentialité qui s'organise autour d'un processus autonome de création de sens. Le couplage acteur-environnement fait émerger la situation dans laquelle l'acteur construit le sens en mobilisant ses références situées, de façon compatible avec le maintien de l'équilibre des structures internes au couplage. Il délimite également de façon autonome le domaine d'interactions possibles avec l'environnement, dans la mesure où il sélectionne les offres de l'environnement en fonction de leur pertinence significative et en relation avec sa clôture opérationnelle. Les savoirs construits à travers cette dynamique d'interactions asymétriques propres au couplage acteur-environnement résultent de l'identification d'un faisceau de ressemblances/différences entre diverses expériences typicalisées.

7 La situation d'alloconfrontation en vidéoformation a la particularité de créer un couplage dans lequel l'acteur interagit avec la situation en partie dévoilée d'un pair. L'activité de compréhension et d'interprétation de l'activité de cet autrui potentialise le processus de reconnaissance : l'observateur.trice éprouve de la familiarité/étrangeté vis-à-vis de la situation d'autrui relativement à ses propres expériences typicalisées, ouvrant sur la reconnaissance d'une similarité entre des actions antérieurement menées et les actions observées. Les propos d'une enseignante-stagiaire confrontée aux commentaires d'une paire dans une vidéo, illustrent ce processus d'auto-référentialité et de reconnaissance : «C'est une situation qui me parle, en fait, ça peut m'arriver heu, ben suivant le contexte, la classe, aussi mon état de fatigue ou mon humeur, de me mettre dans une position comme ça où je vais plus parler». Ce processus de typicalisation explique, pour partie, les possibilités de transfert des savoirs propres de l'acteur d'une situation à l'autre.

8 Ce qui définit l'appartenance à une même catégorie de situations, c'est un gradient de typicité par rapport à un ou des exemplaires prototypiques (Rosch, 1973), ou un air de famille entre les éléments de la catégorie (Kleiber, 1991). Ainsi dans l'exemple pris, l'enseignante fait ce rapprochement entre les deux expériences de classe alors que les vécus des deux enseignantes sont différents : «Par contre, ce que je fais différemment, en fait c'est que, bon éventuellement les bras croisés, ça peut montrer aux élèves : "bon là, j'en ai marre", mais rester immobile complètement et figé au niveau du regard, en fait, je pense que ça les intimide pas en fait et ils sentent qu'on est un peu en retrait et pas présent. Donc, ce que je fais, moi, c'est je peux être dans cette position et me déplacer ou alors balayer du regard la classe et fixer un élève... ». Un peu plus tard, elle se projettera circulant dans sa classe pour ne pas donner une image négative aux élèves. Apprendre dans une situation d'alloconfrontation consiste donc d'une part à répondre aux perturbations produites par l'observation d'autrui (en stabilisant des 
interprétations et des types ou en créant de nouveaux types), et d'autre part à projeter des transformations jugées possibles et réalisables de ses façons de percevoir et d'agir dans la situation cible. Les savoirs construits au cours de ces interactions portent l'empreinte des différents couplages acteur-environnement qui sont mis en parallèle (la situation observée, la situation rappelée, la situation future imaginée). À cette première hypothèse d'autopoï̀se et de typicalisation, nous articulons une deuxième hypothèse renvoyant aux notions de mimésis et de fiction.

\subsection{Mimésis et fiction}

9 En nous appuyant sur les travaux d'anthropologie historique et culturelle de Wulf (2007) relatifs à la mimésis sociale, nous considérons que nous apprenons un acte social " par mimesis, dans un rapport avec d'autres individus que nous voyons agir et auxquels nous sommes liés par le contexte social. Nous vivons la manière dont ils mettent en scène et représentent leurs actes, nous prenons part à leurs actions, nous faisons l'expérience des buts qu'ils poursuivent, du sens qu'ils donnent à ce qu'ils font et du rapport de leurs comportements avec le contexte » (Ibid., p. 9).

Les situations de formation étudiées étant systématiquement marquées par la référence à autrui (confrontation à des vidéos en direct ou en différé du travail d'autrui), elles sollicitent fortement ces processus mimétiques. L'incorporation du monde social à son propre monde peut se réaliser «soit par la participation directe aux actes sociaux, soit par le biais de l'imagination" (Ibid., p.11). Nous défendons donc l'idée que l'apprentissage-développement en formation implique une "expérience mimétique et fictionnelle » (Durand, 2009) qui s'appuie sur l'hypothèse générale développée par Schaeffer (2002) : «dans certaines situations, l'imaginaire, non seulement ne nous empêche pas d'atteindre le réel, mais est une condition indispensable pour l'atteindre " (p. 1). Les situations de formation, notamment lorsqu'elles visent à établir un rapport étroit avec l'environnement de travail, comportent nécessairement une composante fictionnelle active (Durand, 2008; Pastré, 2005), qui peut être plus ou moins forte en fonction de la nature des situations mises en place : simulation, analyse de pratiques, vidéoformation, jeu de rôles, etc. Ces «mises en situation dynamique » favorisent une immersion mimétique qui sollicite à la fois des processus d'identification, de recherche et de production de ressemblances entre des objets, des phénomènes et des activités, par la comparaison de situations vécues passées et présentes et, en même temps, des processus d'anticipation et de projection dans une situation future. Cette recherche de ressemblance, d'airs de famille, ne sert pas à réaliser une copie exacte du réel (tout acte social restant unique), mais à prendre une "empreinte» des actions sociales antérieures qui permet de « créer un rapport à un autre monde » (Wulf, 2007, p. 10).

11 Les « amorces mimétiques » soit sous forme de « semblants quasi-perceptifs » à travers des extraits de films enregistrés ou un film en direct, soit sous forme de «semblants verbaux » (Schaeffer, 1999), à travers des commentaires des acteurs, permettent aux formés de jouer de manière imaginaire dans deux ordres d'activité : d'un côté, celui de la réalité de l'activité professionnelle à travers la confrontation à des expériences significatives faisant écho à leur propre vécu et préoccupations; et de l'autre, celui de l'environnement de formation favorable à des déplacements professionnels fictifs et réflexifs pouvant déboucher sur des possibles d'action jugés transférables dans le réel. 
12 Ainsi, l'univers créé par la confrontation à des extraits filmiques enregistrés ou en direct ne se contente pas de solliciter un processus d'imitation et de «faux-semblant ", mais constitue réellement un vecteur d'apprentissage-développement en offrant la possibilité de construire un certain nombre de savoirs. En effet, les processus mimétiques "ne produisent pas de simples copies de mondes symboliques antérieurs ", mais engendrent un "travail d'appropriation et d'incorporation » conférant à ces relations mimétiques « un aspect créatif » modifiant « sans cesse les mondes originaux de référence " (Wulf, 2007, p. 64). Ces savoirs mimétiques construits sont avant tout « de nature corporelle et étroitement liés à des processus sensibles» (Ibid., p. 11). Les formés transposent ce qu'ils perçoivent et ressentent la manière d'agir d'autrui dans une scène sociale particulière, " par les sens et l'imagination en images intérieures, en mondes du son, du toucher, de l'odorat et du goût qu'ils incorporent à leur propre monde » (Ibid., p. 11).

13 Nous formulons l'hypothèse que la prise en compte de ces processus mimétiques permet de donner un rôle central à la performativité corporelle non prise en compte dans les théories de l'action focalisées sur les seuls aspects cognitifs. Les amorces mimétiques constituées par l'activité d'autrui (manifeste ou implicite) en situation professionnelle (réelle ou simulée) favorisent l'engagement des formés dans la posture $\mathrm{du}$ « faire comme si », en leur permettant de se glisser dans un univers fictionnel. Ces immersions mimétiques provoqueraient un « remodelage de l'expérience » du fait de la mise en résonance d'une double dynamique ${ }^{4}$, reliant des expériences passées vécues en situation de travail avec des expériences vécues lors de la confrontation à l'activité d'autrui (Bouchot \& Leblanc, 2019; Leblanc, 2014 ; Ria \& Leblanc, 2011). Cela soulève, dans le prolongement, la question des méthodes permettant d'appréhender et d'analyser ces opportunités de développement de l'activité, qui émergent à travers l'articulation dynamique, invisible et silencieuse, de ces différents registres d'expérience.

\section{Documenter ces processus mimétiques et fictionnels dans trois contextes de formation professionnelle}

14 Les trois situations de vidéoformation présentées dans cet article sont de natures différentes, que ce soit du point de vue de la sélection et du cumul des méthodes, ou du point de vue des techniques d'entretien utilisées en lien avec les hypothèses de connaissance relatives aux processus mimétique et fictionnel.

\subsection{Autoformation d'enseignant.e.s dans un espace de vidéoformation}

La première situation d'autoformation se déroule dans un espace de vidéoformation qui porte sur l'activité ordinaire d'enseignants débutants. Elle est menée en parallèle de l'activité réelle au travail (formation des enseignants). Des sessions de travail avec la plateforme Néopass@action ${ }^{5}$ ont été réalisées par des enseignantes-stagiaires en Français et en Mathématiques (Leblanc, 2014 ; 2015; Ria \& Leblanc, 2011; Leblanc \& Sève, 2012). En même temps qu'elles naviguaient librement sur la plateforme en 
présence du chercheur, elles étaient invitées à produire des «verbalisations simultanées et interruptives» (Theureau, 2004) lors d'entretiens filmés d'une durée d'une heure chacun (Figure 1).

Figure 1 : Verbalisations simultanées et interruptives.

Figure 1 : Interruptive and simultaneous verbalisations

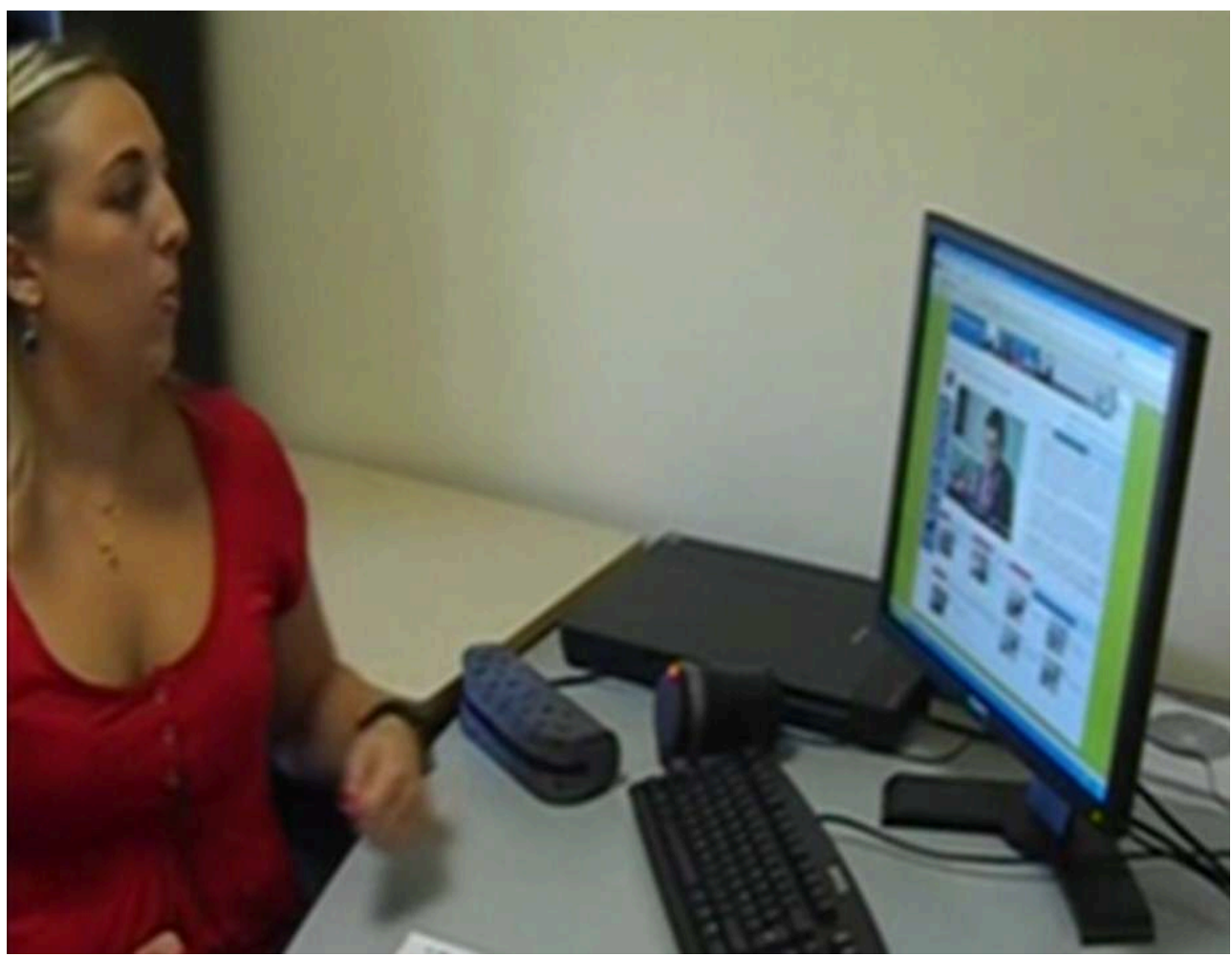

Les données de verbalisation ont été produites par des questionnements et relances du chercheur-interlocuteur qui invitait l'enseignante, tout au long de sa navigation, à expliciter son activité dans la plateforme. Ses relances visaient à faciliter: a) la description de sa « conscience préréflexive » lors de sa navigation, b) l'explicitation du point de vue adopté sur la situation (celui de l'enseignant, des élèves, d'un élève, ou d'elle-même en tant qu'enseignante), c) l'engagement et le maintien dans cette situation de formation, et d) l'énoncé de pistes concrètes qu'elle envisageait de tester dans sa classe (auto-prescriptions). Lors de la session de travail suivante (espacée d'environ un mois), l'entretien démarrait par l'explicitation de ce qui avait été effectivement mis en place en classe à partir des auto-prescriptions construites lors de la session précédente. Dans le cas d'une non-mise en œuvre, il était demandé d'en expliquer les raisons. Ces différentes méthodes ont été articulées pour saisir et comprendre les transformations engagées ou abandonnées par l'enseignante.

\subsection{Observation de sages-femmes en formation lors de séances de simulation haute-fidélité retransmises en direct sur grand écran}

17 La deuxième situation est une situation d'observation de pairs médiée par un grand écran lors de séances de simulation haute-fidélité (SHF) menées en direct sur des cas extraordinaires (formation des sages-femmes). L'étude s'intéresse à l'activité d'observation de cinq étudiantes sages-femmes de quatrième année, inscrites à un 
module optionnel de SHF nommé «Urgences obstétrico-pédiatriques» (Bouchot \& Leblanc, 2019). Cinq séances sont réparties sur le premier semestre 2016. Chaque étudiante se porte volontaire pour être actrice de deux situations simulées, une fois en qualité de sage-femme et une fois en qualité d'aide-soignante. Elles sont observatrices lors des trois autres séances. La méthode a consisté à réaliser des entretiens d'autoconfrontation sur la base des multiples traces de l'activité : l'enregistrement de l'écran de projection à quatre entrées restituant en direct la simulation réalisée par des pairs, l'enregistrement audio-vidéo de plusieurs observatrices (filmées de face), les traces de l'activité d'écriture accompagnant l'observation (Figures 2 et 3).

Chaque entretien a sollicité l'expression de la conscience préréflexive des observatrices grâce à une remise en situation dynamique rendue possible d'une part, par les diverses traces de leur propre activité, et d'autre part par les formulations des questions du chercheur (Theureau, 2011). Les conditions matérielles et dialogiques retenues visaient à dé-situer l'observatrice de la situation présente, à la re-situer dans la situation étudiée et à l'y maintenir pour accéder aux significations construites pas-à-pas. La méthode ne visait pas de nouvelles prises de conscience, au contraire elle cherchait à les éviter. La conduite des entretiens consistait tant que possible à proscrire les questions pouvant sortir les observatrices du vécu ou revécu de l'activité à laquelle elles étaient confrontées (pourquoi ?), à employer des relances ou questions vides de contenu (et là ?) ou encore à les resituer ici et maintenant par l'emploi du présent ( $L a ̀$, vous écrivez...). Au total, 13 entretiens d'autoconfrontation ont été réalisés.

Figure 2 : Données documentant l'activité manifeste des observatrices.

Figure 2: Data documenting the observers' overt activity

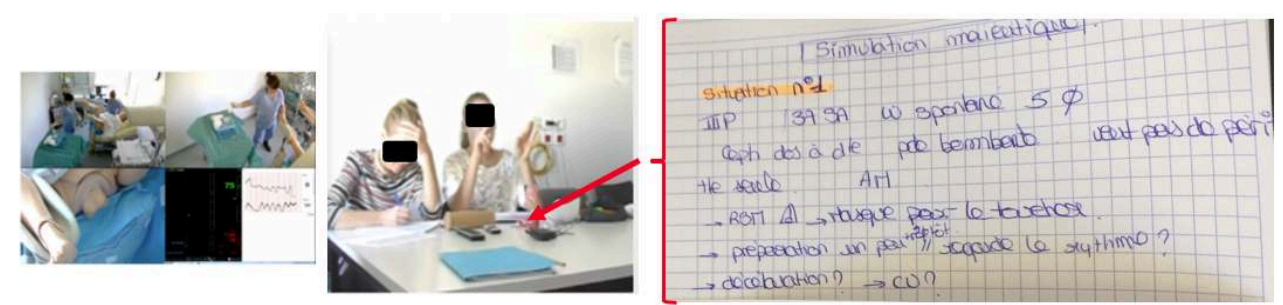

De gauche à droite : situation de simulation observée / enregistrement audio-vidéo des observatrices / traces de l'activité d'écriture

Left to right: Observed simulation situation / Two observers' audio-video recording / Traces of the writing activity

Figure 3 : Entretien d'autoconfrontation simple d'une observatrice aux traces de son activité. Figure 3: Simple self-confrontation interview of an observer to the traces of her activity
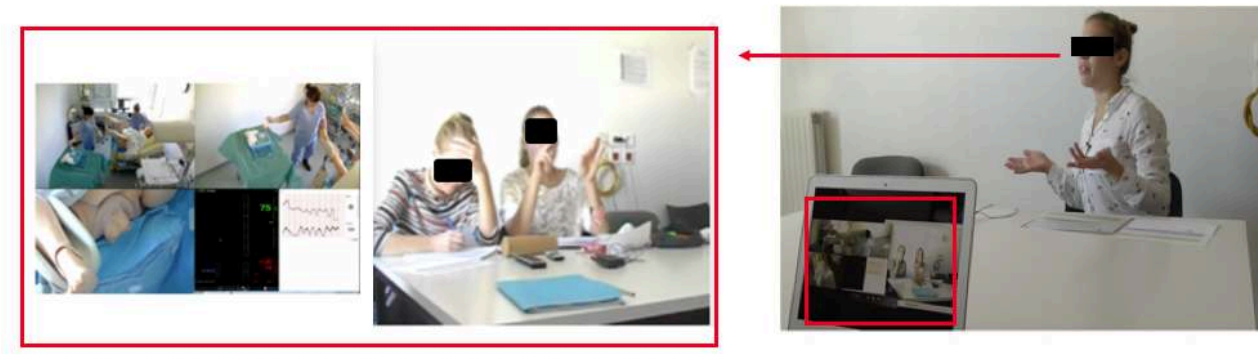


\subsection{Autoformation dans un espace de vidéoformation et de simulation à la conduite de chevaux attelés} minutes, vécue par une stagiaire dans un espace de vidéoformation à la conduite de chevaux attelés ${ }^{6}$. Ce dispositif de vidéoformation, nouveau dans l'environnement de formation ${ }^{7}$, a été présenté aux stagiaires dès le début de la formation en leur laissant la possibilité de l'utiliser en autonomie en se connectant avec leurs identifiants. Deux mois après le début de la formation, au moment où se déroule la situation étudiée, les stagiaires n'avaient pas encore utilisé cet outil avec leur formateur. Par ailleurs, la chercheuse, en immersion ethnographique sur la totalité de la formation, est identifiée par les formés comme une stagiaire particulière faisant une recherche sur leur activité. Nadège, la stagiaire concernée par cette étude, a déjà réalisé un entretien d'autoconfrontation sur une situation de conduite au cours du premier mois de formation. Elle a aussi participé à des films ethnographiques pendant lesquels la chercheuse a enregistré son activité de conduite. En revanche, elle n'a pas exploré la plateforme de vidéoformation. La participation à cette situation d'autoformation a été négociée entre la chercheuse et la stagiaire. Cette négociation a débouché sur l'accord suivant : la chercheuse s'engage à accompagner la stagiaire dans la découverte de cette plateforme de vidéoformation, en lui laissant la main sur la navigation, et, en contrepartie, la stagiaire accepte de consacrer une part de son temps libre à la chercheuse, afin qu'elle puisse construire des données de recherche sur son activité de navigation/visionnage dans le dispositif d'autoformation.

Les vidéos de cette plateforme ont la particularité de porter sur l'activité ordinaire de meneurs expérimentés. Cependant, au moment de sa découverte, la stagiaire n'a pas connaissance de l'expertise de ces meneurs d'attelage.

Lors de la situation d'autoformation retenue, l'entretien négocié avec la stagiaire propose d'articuler le « dire à la chercheuse ce que je suis en train de faire » sur la base $\mathrm{du}$ " penser tout haut ", et des relances de la chercheuse sur la base des verbalisations simultanées interruptives (comme pour l'étude 1 de la situation de l'enseignante). Cette articulation prend en compte les vigilances à avoir vis-à-vis du maintien dans l'activité de navigation/visionnage afin de documenter au plus près la conscience préréflexive de la stagiaire. De la même manière, la chercheuse a veillé à intervenir à des moments qu'elle jugeait opportuns afin de garantir la dynamique de l'activité en cours.

Cette situation de navigation/visionnage médiée par la chercheuse a été le point de départ d'une transformation significative pour la stagiaire, par l'émergence d'une nouveauté dans son «monde, corps et culture» propre: "l'histoire du poids" (voir 5.2.2.). Cette transformation qui apparait dans cette situation de visionnage se prolonge dans les jours suivants et accompagne la stagiaire jusqu'à la fin de sa formation. La chercheuse a reconstruit cette transformation pour cette stagiaire en mobilisant diverses données ethnographiques, les verbalisations en entretien portant sur son activité en situation de conduite, sur son activité de visionnage et de simulation de manipulation des guides, ainsi qu'un entretien invitant la stagiaire à porter un regard rétrospectif sur sa formation.

La frise chronologique (Figure 4) donne à voir l'ensemble des données construites spécifiquement avec cette stagiaire. Le marquage vert indique la situation présentée dans cet article. 
Figure 4 : Frise chronologique des données construites avec la stagiaire Nadège. Figure 4: Timeline of the data built with the trainee Nadège

\begin{tabular}{|c|c|c|c|c|c|c|c|c|c|c|c|}
\hline & \multicolumn{2}{|c|}{ Période 1} & \multicolumn{4}{|c|}{ Période 2} & \multicolumn{5}{|c|}{ Période 3} \\
\hline $\begin{array}{l}\text { Date } \\
\text { Activité }\end{array}$ & $\begin{array}{l}23.11 \\
\text { En } \\
\text { voiture }\end{array}$ & $\begin{array}{l}04.12 \\
\text { En } \\
\text { voiture }\end{array}$ & $\begin{array}{l}03.01 \\
\text { En } \\
\text { voiture }\end{array}$ & $\begin{array}{l}09.01 \\
\text { Sur } \\
\text { Ercam }\end{array}$ & $\begin{array}{l}10.01 \\
\text { Discus- } \\
\text { sion }\end{array}$ & $\begin{array}{l}11.01 \\
\text { Discus- } \\
\text { sion }\end{array}$ & $\begin{array}{l}19.02 \\
\text { Sur } \\
\text { Ercam }\end{array}$ & $\begin{array}{l}13.03 \\
\text { En } \\
\text { voiture }\end{array}$ & $\begin{array}{l}14.03 \\
\text { En } \\
\text { voiture }\end{array}$ & $\begin{array}{l}22.03 \\
\text { Sur } \\
\text { Ercam }\end{array}$ & $\begin{array}{l}26.03 \\
\text { CoMtacts }\end{array}$ \\
\hline $\begin{array}{l}\text { Traces } \\
\text { de } \\
\text { l'activité }\end{array}$ & & foryes & & $\sigma^{2}$ & & & & & & & \\
\hline $\begin{array}{l}\text { Verbali } \\
\text {-sations }\end{array}$ & $3 \frac{5}{7}$ & & & & & & & $x 1$ & & & \\
\hline $\begin{array}{l}\text { Cours } \\
\text { de T.A }\end{array}$ & & $13^{\circ}$ & $17^{\circ}$ & & $20^{\circ}$ & & $22^{\circ}$ & $28^{e}$ & $29^{\circ}$ & & \\
\hline
\end{tabular}

\section{Traduction théorique des processus mimétiques}

Pour approfondir la compréhension des processus mimétiques à l'œuvre dans ces différentes situations de formation, nous proposons une contribution théorique au programme de recherche du cours d'action, en ouvrant des pistes d'analyse pour mieux saisir les dimensions implicites de l'activité corporelle et sensible, très présentes dans ce rapport mimétique au monde d'autrui, et permettre de mieux comprendre les conditions de performativité ${ }^{8}$ de l'action.

\subsection{L'hexatomie de l'unité d'expérience}

Un cours d'expérience est composé d'un enchaînement d'unités d'activité significatives pour l'acteur, ou unités de cours d'expérience. Chacune de ces unités émerge d'un signe hexadique reliant six composantes (Figure 5) rendant compte des processus essentiels de la construction de l'expérience humaine ${ }^{9}$. Les trois premières composantes constituent la structure d'attente de l'acteur, qui délimite le champ des possibles de l'activité à l'instant $\mathrm{t}$ (Engagement dans la situation ; Actualité potentielle ou Structure d'anticipation; Référentiel). Les deux composantes suivantes constituent l'actualité de l'acteur à l'instant $t$ (Representamen ; Unité de cours d'expérience). Enfin, la dernière composante traduit la construction de savoir inhérente à l'unité de cours d'expérience à l'instant $\mathrm{t}$ (Interprétant). Ce qui est le plus actuel pour l'acteur correspond à l'unité de cours d'expérience (ou unité significative) $U$ en relation avec un Representamen $R$. Cette unité décrit l'expérience de l'acteur à l'instant $t$ en lien avec son activité, qui peut être montrée - voire mimée - et mise en mots. Elle traduit la plupart du temps une action (Pratique ou de communication) ou un sentiment. 
Figure 5 : Signe hexadique.

Figure 5 : Hexadic sign

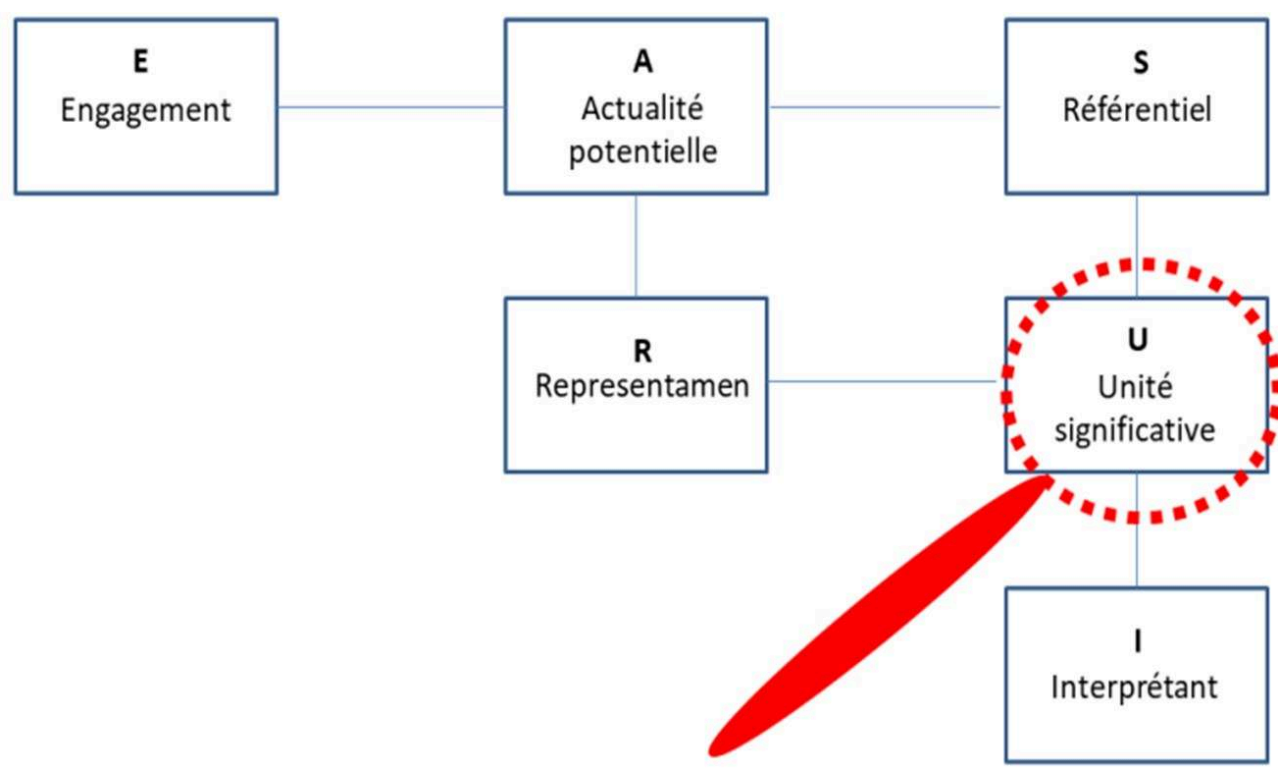

Pour mieux rendre compte des processus mimétiques (immersion, imitation, émulation, "faire le semblable de", représentation, anticipation) et de la performativité corporelle évoqués précédemment, nous explorons et exploitons le potentiel de l'hexatomie de l'unité d'expérience $\mathrm{U}$ qui offre des possibilités de description nouvelles de l'activité à des grains très fins ${ }^{10}$, à partir de sous-composantes abstraites proposées par Theureau (2006, 2009). L'hexatomie du U (Figure 6) s'organise sur la base des mêmes relations réelles ${ }^{11}$ et de pensée ${ }^{12}$ que le signe hexadique. Cela signifie que si le chercheur voit apparaître une action ou imagination [U.2.2] dans l'expérience de l'acteur, cette dernière est par hypothèse consécutive à une impulsion [U.1.1] et à l'émergence d'un sentiment [U.2.1]. 
Figure 6 : Hexatomie de l'unité d'expérience. Figure 6 : Hexatomy of the experience unit

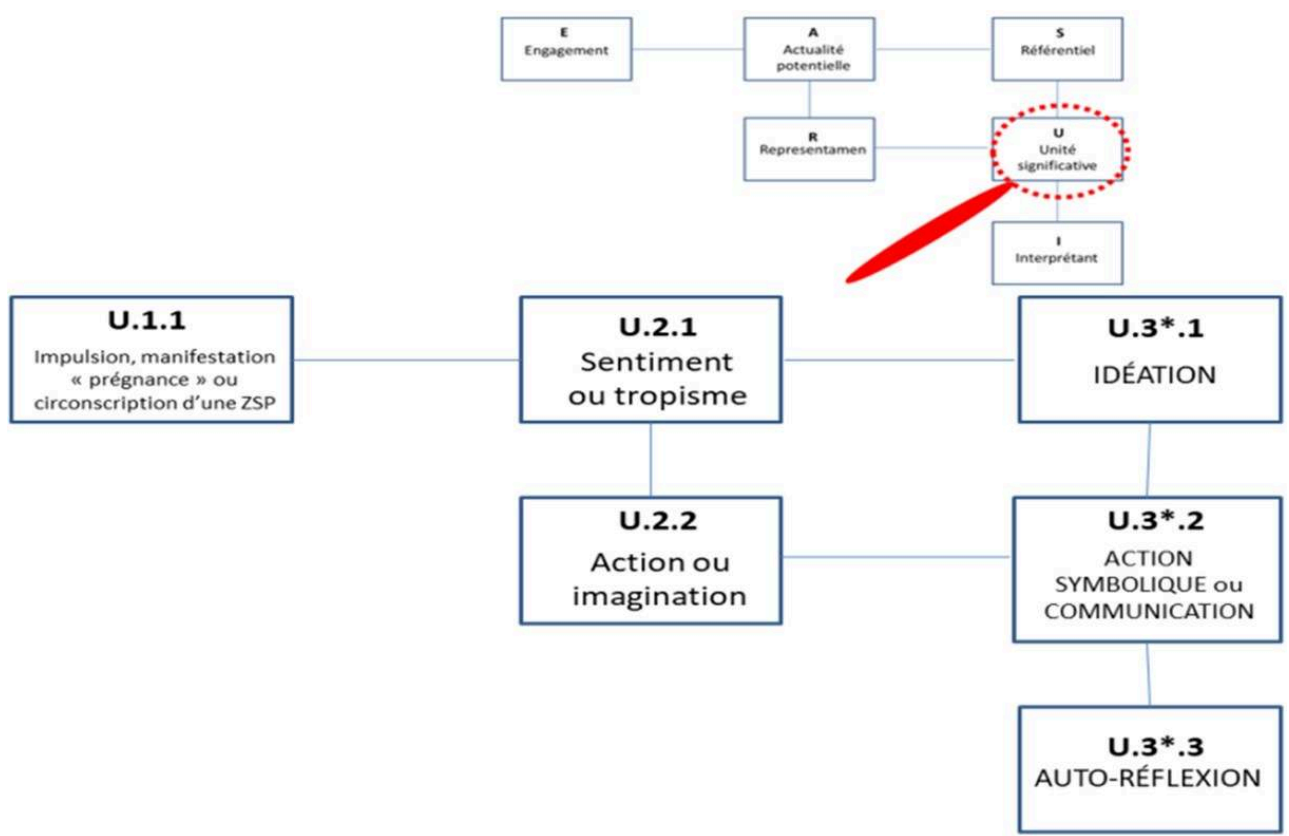

Cet aspect nous paraît important, car les U relevant d'un caractère dégénéré c'est-àdire se terminant dans la déclinaison de l'hexatomie par un .1, s'accompagnent d'une part d'indétermination ou de vague, en raison de leur caractère premier (i.e. de leur caractère de Priméité ou Possible). En cela, ils sont difficiles à déceler à la fois pour l'observateur extérieur et pour l'acteur. Pour pouvoir les documenter, l'acteur doit reconnecter avec son vécu et doit explorer des moments flous, fugaces, difficiles à mettre en mot.

Le cadre méthodologique du « cours d'action » permet de renseigner la dynamique de l'activité en documentant-décrivant la conscience préréflexive attachée à la situation (notamment à partir des méthodes de verbalisation simultanée et d'autoconfrontation). Il permet également de saisir des détails sensoriels et émotionnels de l'action notamment à partir d'une remise en situation par les traces laissées dans le corps des acteurs. Toute la difficulté méthodologique réside dans l'articulation de cette double exploration à la fois celle de la dynamique temporelle et culturelle de l'activité (sur des empans plus ou moins grands) et en même temps celle de l'expérience sensible en profondeur de l'ici et maintenant. Pour avancer sur cette question difficile, quelques chercheurs (e.g. Azéma, 2015 ; Azéma, Secheppet, \& Mottaz, 2020 ; Cahour, 2006) ont réalisé des rapprochements théoriques et assemblages méthodologiques issus de l'anthropologie cognitive, de l'ethnographie et de la psycho-phénoménologie. Pour soutenir l'hypothèse sur la fonction mimétique, nous montrons plutôt comment traiter des données en raffinant les catégories d'analyse qui permettent de formaliser les transformations en cours (impulsion, sentiment, imagination...) que la manière de les construire sur le terrain et en collaboration avec les acteurs.

\subsection{Analyse de l'expérience mimétique à partir de l'hexatomie du U}

Pour illustrer le type de déconstruction-reconstruction de l'expérience mimétique à partir de l'hexatomie du U et préciser les définitions des sous-catégories, nous nous 
appuyons sur l'étude de cas 1 réalisée avec une enseignante-stagiaire qui est venue naviguer librement sur la plateforme Néopass@ction (une fois par mois durant 4 mois) en présence du chercheur (Leblanc, 2014). Nathalie (professeure de Mathématiques) se retrouve à écouter les propos d'une enseignante néotitulaire d'Éducation Physique et Sportive (EPS) (nommée Aude) qui commente et réagit à la vidéo d'un enseignant en difficulté (nommé Romain) dans son début de cours. Nathalie va être attirée par l'histoire des «bras croisés » à partir des propos d'Aude en écho à sa propre pratique (Figure 7). Elle poursuivra cette histoire en arrêtant la vidéo sur la position de l'enseignant Romain qui se tient les « bras croisés » contre le tableau.

Figure 7 : Enchaînement de deux signes sur l'histoire des «bras croisés ".

Figure 7: Series of two signs concerning the "crossed arms" story

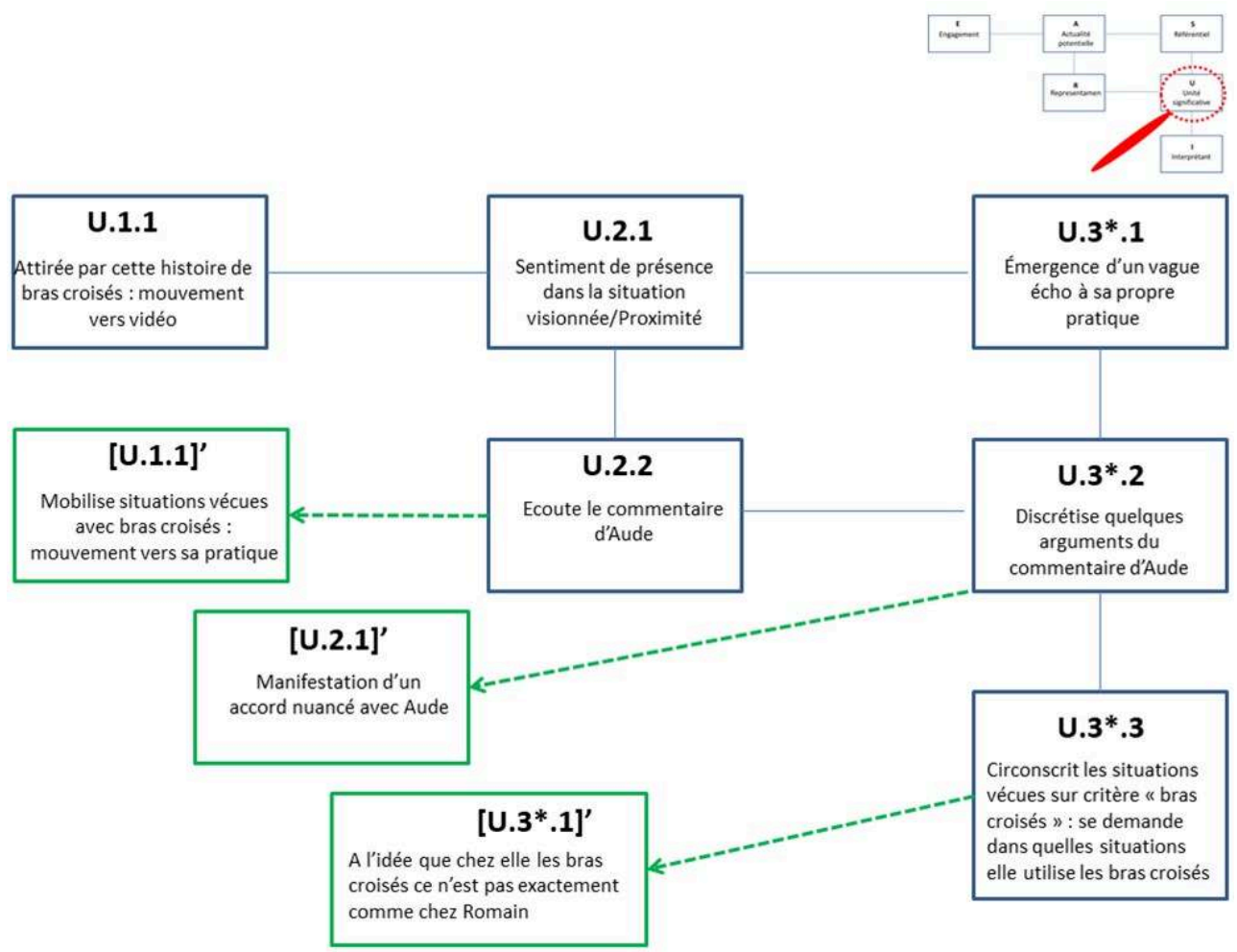




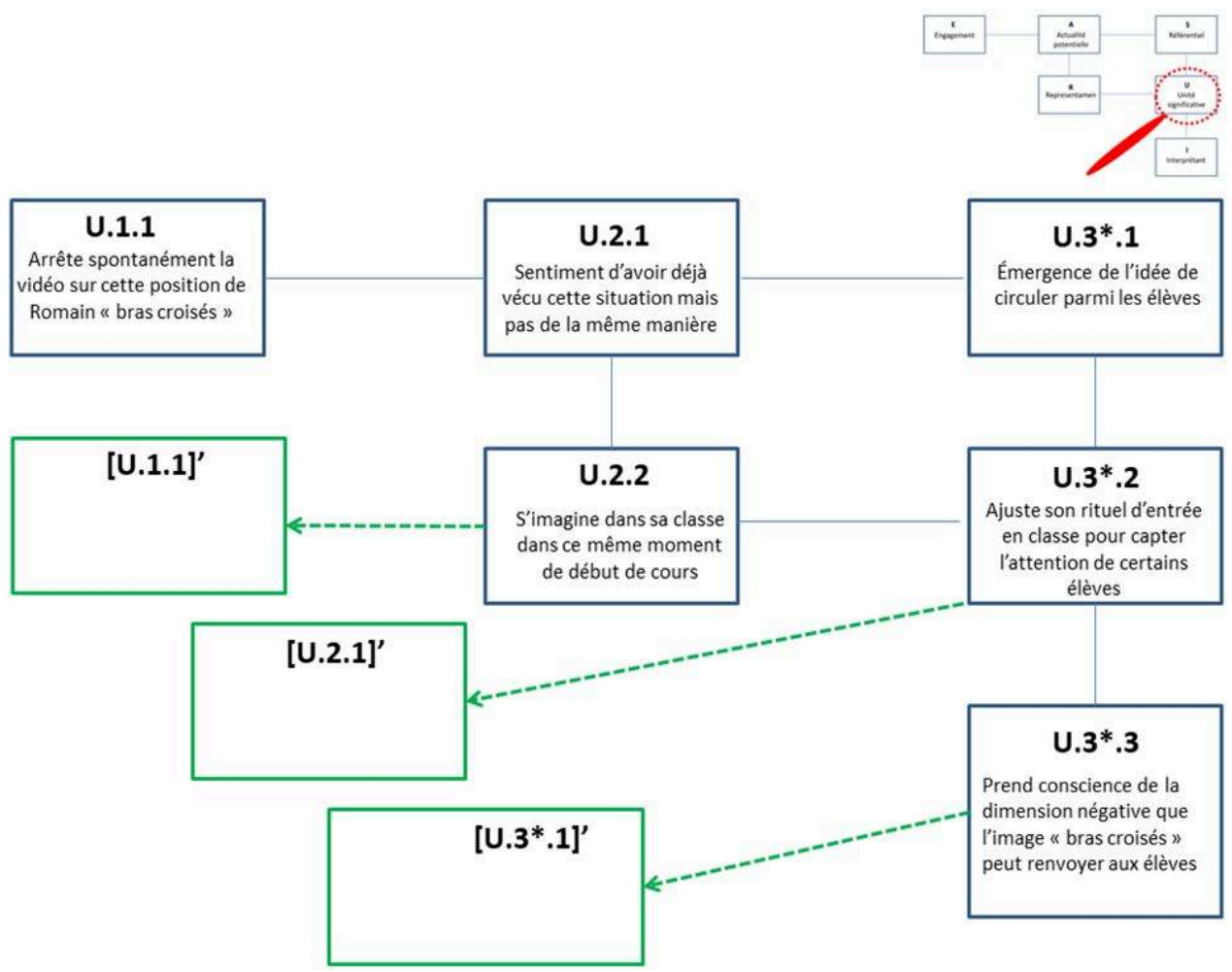

Toute apparition d'une unité d'expérience $[\mathrm{U}]$ se fait en lien avec une impulsion (notée [U.1.1]), c'est-à-dire une force psychique, affective, nerveuse, sensuelle, aveugle, intérieure, irraisonnée, irréfléchie, obscure, secrète, soudaine, subite, violente et spontanée, qui s'impose à la perception sans contrôle possible et pousse à l'action. Pendant l'écoute de la vidéo, Nathalie est très attentive et proche de l'écran [U.1.1]. À l'issue de l'écoute [U.2.2], elle manifeste spontanément et immédiatement un certain « accord » avec les propos d'Aude concernant le fait de « rester les bras croisés, sur une même posture » [U.2.1]'. Elle reproduit spontanément la position des bras croisés en la mimant [U.1.1] sur la base de ce qui a fait «choc» pour elle dans les propos de l'enseignante d'EPS : «bon éventuellement les bras croisés, ça peut montrer aux élèves "bon là, j'en ai marre" » (Figure 8). Son mouvement de pensée l'amène à se positionner instantanément au sujet de cette posture, qui fait écho à sa pratique [U.3*.1]. 
Figure 8 : Mime les bras croisés. Figure 8 : Mimes the crossed arms

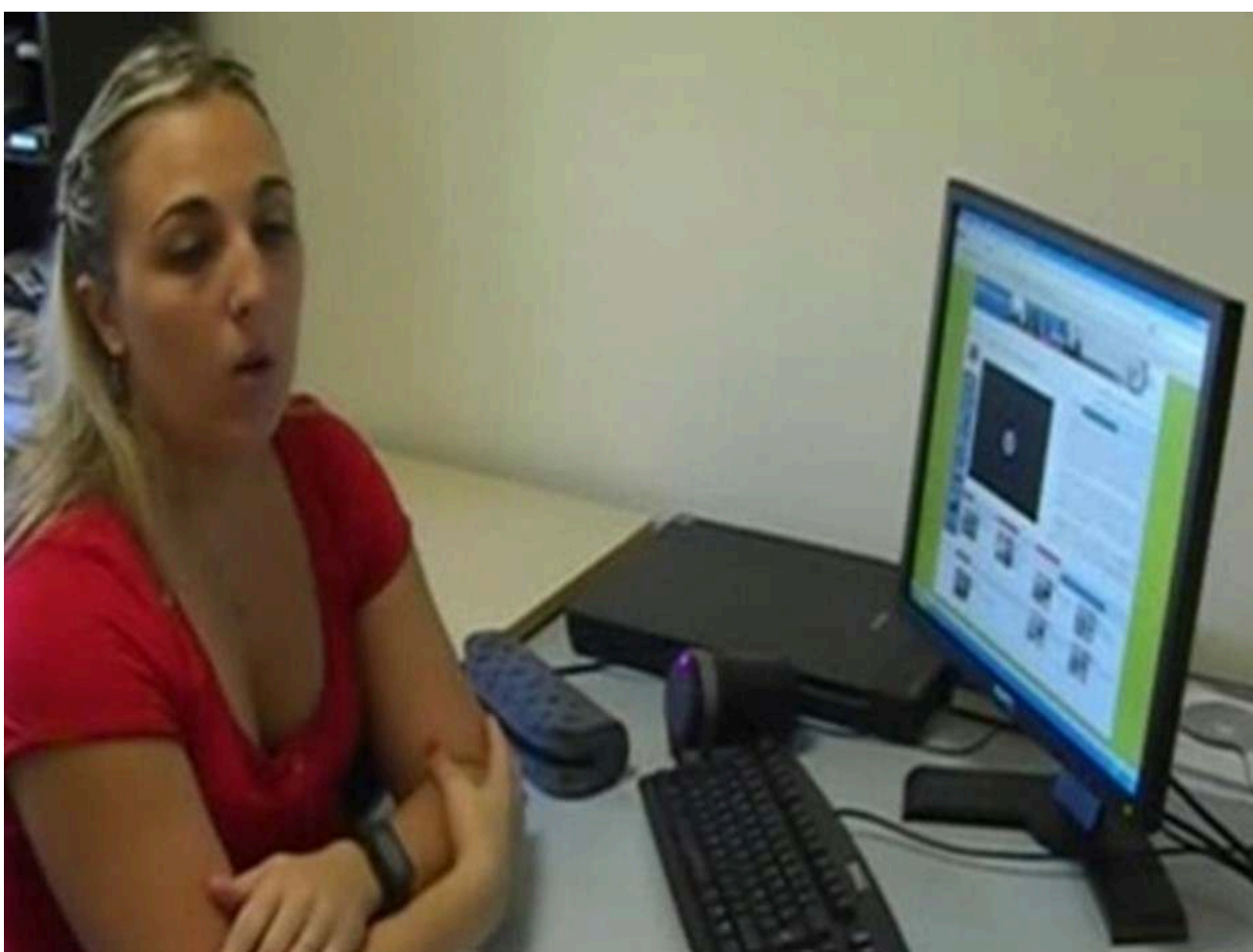

La manifestation d'une prégnance se manifeste également dans le corps qui est un « espace expressif» «où, imperceptiblement, le mouvement se fait geste, le geste se fait signe, le signe parole, sans que l'on puisse repérer où s'arrête le mouvement brut et où commence le signe" (de Saint Aubert, 2010, p. 135). Ainsi, Nathalie mime un balayage du regard en faisant des mouvements de tête et de bras de droite à gauche (Figure 9) tout en disant "ça marche, mais à condition de soit balayer la, comme elle dit, de balayer la classe du regard» [U1.1]. 
Figure 9 : Mime le balayage de la tête avec les bras. Figure 9: Mimes the head sweeping with arms

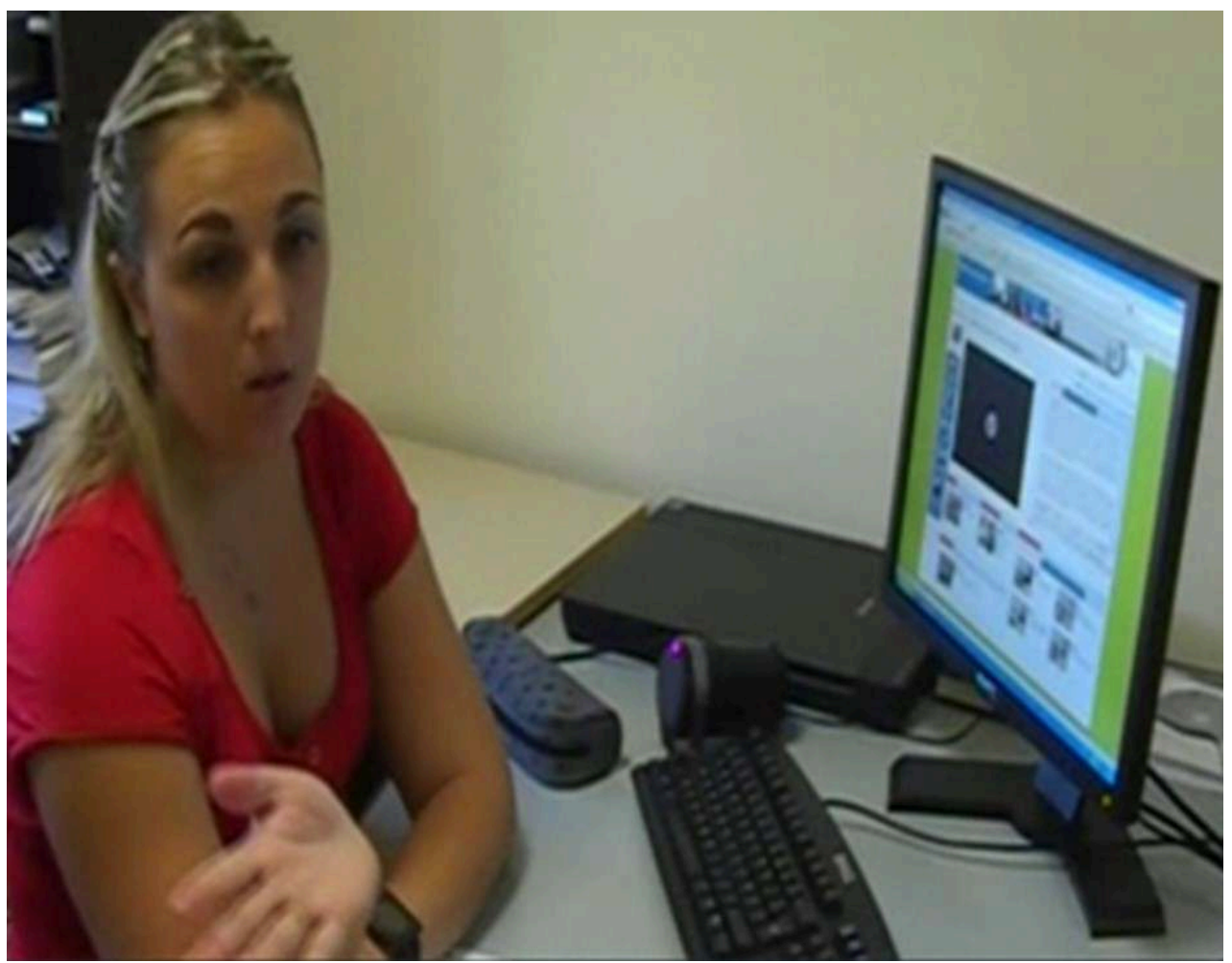

Cette impulsion s'accompagne de tropismes [U.2.1], définis comme des «mouvements indéfinissables, qui glissent très rapidement aux limites de notre conscience " (Sarraute [1996, p. 1719] cité par Theureau, 2006, p. 336) et qui sont à l'origine de nos actions et/ou émotions. Ainsi Nathalie ressent un sentiment de présence [U.2.1] dans la situation fictive due au lien de proximité qu'elle établit avec un vécu de classe. L'enchainement de gestes "iconiques ${ }^{13}$ mimant la posture bras croisés, le balayage du regard et des gestes visant à interpeller des élèves (Figure 9) nous permettent de dire qu'elle entre dans le processus mimétique. Ces éléments nous semblent propices à la poursuite de cette expérience mimétique via une forme de synchronisation entre les dispositions à agir de l'acteur à ce moment-là et la vidéo visionnée. À cet instant, l'acteur compose avec divers « chocs » (Romain : bras croisés en étant figé), « appels » constitués ici par les propositions d'autrui (Aude : bras croisés en étant active), mais aussi «imaginations productrices» (Theureau, 2009, p. 104) qui vont permettre de poursuivre le processus mimétique en s'installant dans la fiction.

Le processus mimétique s'enrichit grâce à une conscientisation des liens établis entre les expériences (en formation et/ou au travail) qui se réalise par l'imagination [U.2.2]. L'imagination productrice se réalise sur la base d'une recomposition et/ou d'articulation d'images mentales à partir d'objets déjà perçus et rappelés. Les images mentales détiennent plusieurs statuts et des modes d'existence différents selon les moments : avant l'expérience pour anticiper (futur), durant celle-ci pour percevoir (présent) et après celle-ci comme symbole-souvenir (passé). Dans le prolongement de l'action de mime d'un balayage du regard, Nathalie évoque, sur la base d'«imagessouvenirs ", une partie de ses expériences qui font écho aux propos d'Aude "Alors, moi je m'attarde aussi sur heu, enfin je regarde précisément les élèves qui sont pas en place pour moi » (Figure 10). 
Figure 10 : Geste iconique qui appuie la précision de son regard.

Figure 10: Iconic gesture that supports the precision of his gaze

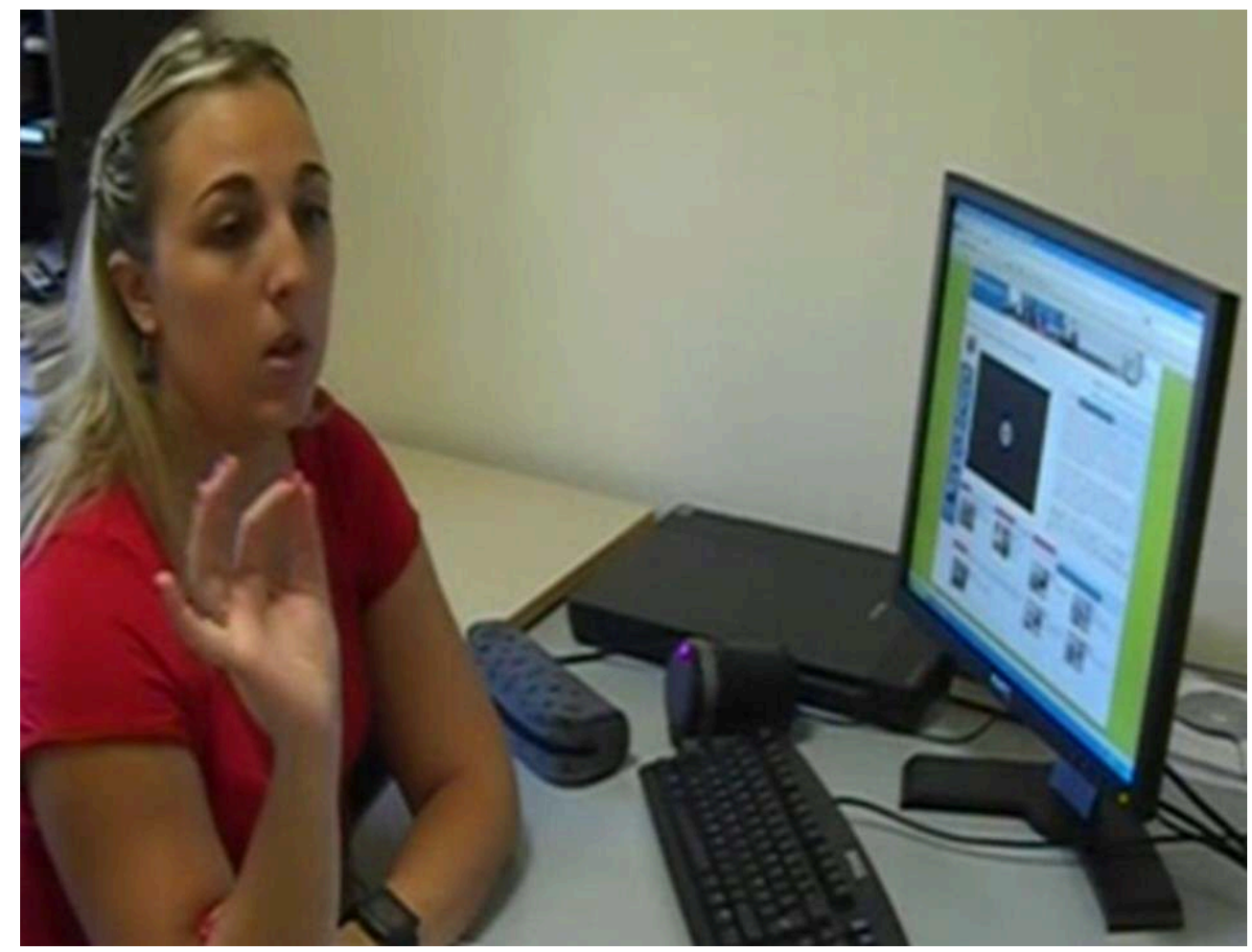

Elle se remémore son rituel d'entrée de classe dans lequel vient s'insérer cette focalisation sur certains élèves : « quand ils entrent en classe en fait, ils sont debout, ils restent debout, ils doivent sortir toutes leurs affaires de maths et ensuite, j'attends le silence pour les faire asseoir ». En imaginant et en rejouant ce fragment d'expérience, elle voit et décrit les comportements inattentifs ou déviants des élèves ainsi ce que cela la conduit à faire : "souvent il y en a, ils sont dans la lune, donc ils oublient de sortir leurs affaires. Donc, je leur rappelle. Ou il y en a qui s'assoient avant que je le leur aie demandé, donc, je leur rappelle aussi ».

L'unité d'expérience peut se traduire aussi par l'émergence d'une idée (idéation) [U.3*. 1] correspondant à ce que l'esprit peut concevoir comme idée approximative, complexe, concrète, confuse, distincte, imparfaite, insuffisante, nette, sommaire, superficielle, vraie, faible, vague, d'ensemble. L'idée qui émerge chez Nathalie à la suite de cette immersion fictive s'exprime tout d'abord de manière assez vague : « Mais par contre, ce que je pense que je vais heu, je vais faire, c'est effectivement ben passer dans la classe ». Cette idée de circuler parmi ses élèves s'est construite sur la base des commentaires d'Aude qui développait dans ses propos la nécessité d'être active pour signifier sa présence et ses intentions aux élèves : « rester immobile complètement et figé au niveau du regard, en fait, je pense que ça ne les intimide pas ", «il faut après qu'il y ait quand même quelque chose qui bouge, soit le regard, soit se déplacer ». Cette idée s'affinera et se confortera en voyant Romain appuyé contre le tableau dans une posture bras croisés [U.3*.1]'.

L'unité d'expérience peut s'exprimer par une action ou communication symbolique [U. $\left.3^{*} .2\right]^{14}$ en tant qu'elle est partagée et donc interprétable par une communauté culturelle. Notons qu'il peut y avoir plusieurs périmètres de communauté culturelle. 
Par exemple, un stagiaire s'inscrit à la fois dans la communauté partagée avec les autres stagiaires, dans celle partagée avec les formateurs, et dans celle partagée avec les tuteurs de stage. Nathalie prend conscience que la manière de mener son rituel : « Je balaye du regard, mais effectivement, heu, comme ils sont debout, des fois je les vois pas tous", ainsi que l'organisation spatiale de sa classe: "moi j'ai un bureau qui est complètement, il est pas au centre, il est tout à droite de la classe, bon avec un ordinateur et c'est vrai que je suis un peu en retrait finalement par rapport à... », ne favorisent pas le contrôle des élèves uniquement par le regard. Ces deux prises de conscience renforcent ainsi l'intérêt d'explorer et de tester des déplacements dans cette phase particulière de préparation des élèves au travail, pendant laquelle elle attend le silence : «justement montrer que ben, et plus facilement en tout cas à ceux qui sont pas en place, ben, de les faire un petit peu heu s'activer... pour qu'ils se mettent plus rapidement en position de travail».Cette vision nouvelle du rituel d'entrée en classe et de la mise en activité des élèves s'est construite par la mise en comparaison de son propre monde et de deux mondes appartenant à autrui, qui entrent en dissonance (la vidéo de Romain en difficulté que Nathalie avait déjà vue/les commentaires d'Aude relatifs à la vidéo de Romain complétés de ses propres façons de faire en lien avec la spécificité de l'EPS). Cette transformation potentielle de son rituel d'entrée en classe est renforcée par une autre comparaison effectuée quelques secondes plus tard, qui va lui permettre une prise distance sur ce qui se joue dans ce type de situation.

Enfin, l'unité d'expérience peut déboucher sur la production d'un discours auto-réflexif qui porte sur le fond de toutes les autres sous-catégories et de leurs transformations [U. 3*.3] (Theureau, 2009). Les jugements issus de cette auto-réflexivité s'expriment toujours à travers un couple par exemple entre « l'agissant et l'agi » (Fichte, 2000, cité par Theureau, 2009). Alors que Nathalie s'apprête à lancer la vidéo de l'entretien dans laquelle on voit Romain en position d'attente les bras croisés, elle commente en se reculant sur sa chaise et en mimant la posture de Romain [U.3*.2] : "Ah, là, il a une posture qui est contre le tableau, il s'appuie comme s'il avait besoin de, de soutien un petit peu. Et ça, on m'avait fait la remarque l'an dernier quand j'avais fait le stage, j'avais un petit peu cette attitude-là... ". Ici, c'est la mise en relation (l'agissant) de la posture contre le tableau de Romain, le rappel du retour d'une formatrice lors d'une visite de classe sur cette attitude (qui n'avait pas fait sens à ce moment-là, un an auparavant) et du vécu associé à celle-ci « [moi] je n'attendais pas de soutien » [U.2.1], qui l'amène à prendre conscience que cette attitude peut renvoyer une image au niveau des élèves (l'agi) en décalage total avec les intentions de l'enseignant, image que l'on peut qualifier de quasi-transparence [U.3*.3] : «finalement, c'est comme s'il s'effaçait un petit peu là, de, de s'appuyer au tableau ». Cette auto-réflexion s'élabore tout d'abord par une mise en rapport de son monde propre et du monde d'autrui (Romain et Aude), par laquelle elle construit d'une certaine façon l'importance de la présence physique et spatiale de l'enseignant.e dans la classe, et ensuite par la comparaison d'une expérience similaire issue de son "passé lointain " (l'année précédente) avec le monde propre de Romain. L'auto-réflexion [U.3*.3] qui s'est déployée à partir de cette séquence a provoqué des transformations réelles au niveau de sa pratique, inspirées directement de ce qu'elle a construit durant la navigation sur la plateforme : « Je ne fais plus l'appel, je circule dans la classe jusqu'à ce qu'ils aient tous sorti leurs affaires et puis après, je les fais asseoir et voilà. Je me suis un petit peu servi de ce que j'avais vu 
justement, plus rester devant le tableau à attendre, mais circuler pour montrer que j'attends qu'ils soient, enfin qu'ils se mettent rapidement en place ».

\section{Mise à l'épreuve de l'élaboration théorique dans deux autres études}

En mobilisant cet outil conceptuel, nous proposons des illustrations documentant certains processus mimétiques dans deux autres contextes de formation : d'une part en formation initiale de la pratique maïeutique par simulation haute-fidélité (étude de cas 2) et, d'autre part, en formation à la conduite de chevaux attelés (étude de cas 3). Le premier contexte de formation a la particularité de faire vivre aux sages-femmes stagiaires, sur un temps court, une situation fictive en petit collectif et en direct. Le deuxième contexte a la particularité de proposer des espaces de formation différents (vidéoformation, simulations, et conduite d'attelage) et de s'inscrire sur du temps long (6 mois). L'intérêt de mobiliser ces deux recherches est la complémentarité qu'elles offrent: la première s'est centrée sur l'activité d'observation en situation de visionnage; la seconde a cherché à pister les transformations entre les différents contextes de formation comprenant des visionnages.

\subsection{Simulation haute-fidélité menée en direct sur des cas extraordinaires}

\subsubsection{Immersion mimétique, enquête et jeu}

39 Le premier moment choisi se situe à l'occasion d'une séance qui vise à développer des compétences techniques et non techniques contribuant à une gestion pertinente de la réanimation du nouveau-né. Pour des raisons organisationnelles, la projection filmique en salle de débriefing est initiée avant que la séance n'ait commencé, c'est-à-dire avant même que les étudiantes actrices de la situation simulée n'entrent en scène. Trois étudiantes sont observatrices dans la séance. Elles anticipent que la situation conçue par les formatrices expose les actrices à devoir résoudre une complication obstétricopédiatrique per natale, sans en connaître la nature: "les scénarios de simulation impliquent de devoir gérer une urgence, des situations inhabituelles et un peu stressantes, ça ne va pas être de la physiologie ». Dès le lancement des quatre entrées vidéo, qui offrent différents plans de l'environnement simulé (Figure 11), elles regardent l'écran télévisé. 
Figure 11 : Projection filmique, à quatre entrées, de l'environnement simulé. Figure 11: Filmic projection with four video inputs of the simulated environment

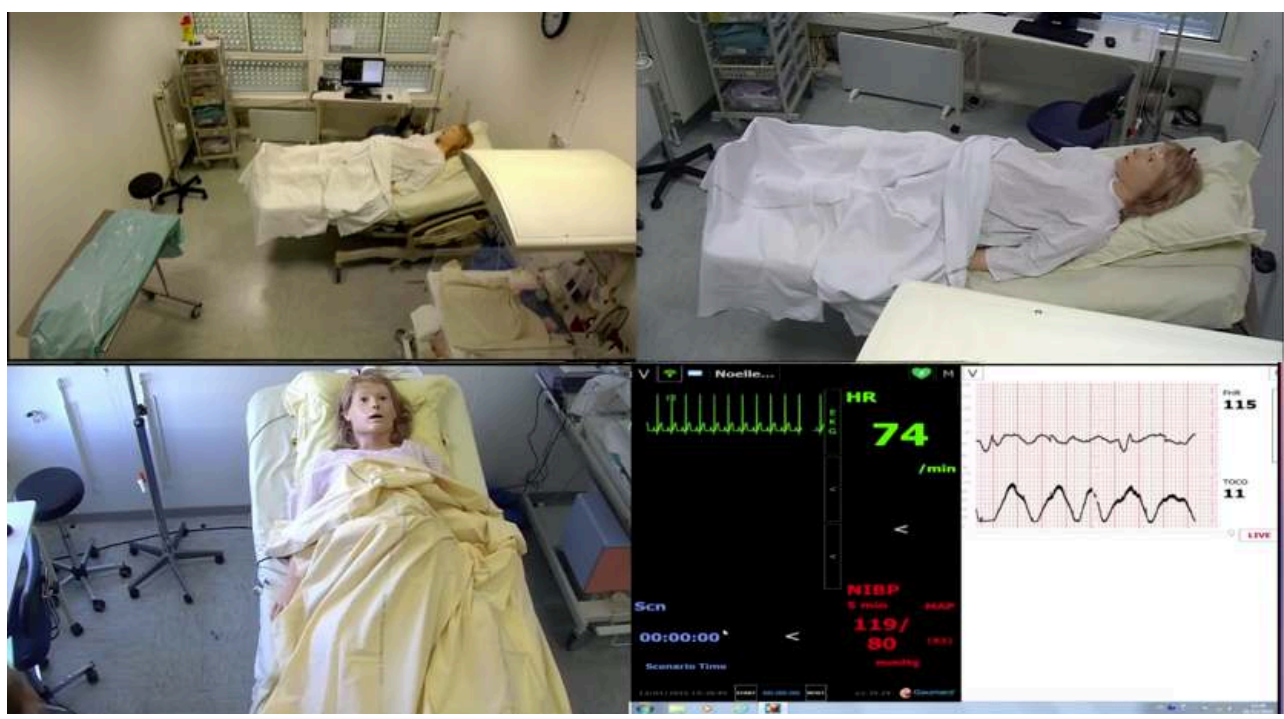

Estelle visualise le rythme cardiaque fœtal (RCF): «là, la télé s'allume et j'ai vu le rythme [...]» [U 2.2]. Elle est bouche bée (impulsion [U 1.1]) devant la découverte du rythme (Figures 12) qui s'impose à sa perception : «il nous fait réagir » (manifestation d'une prégnance [U 1.1]), et s'accompagne d'un état de sidération : « on est choqué ».

Figure 12 : Extrait photographique de l'activité manifeste d'Estelle. Figure 12 : Photographic extract of Estelle's observable activity

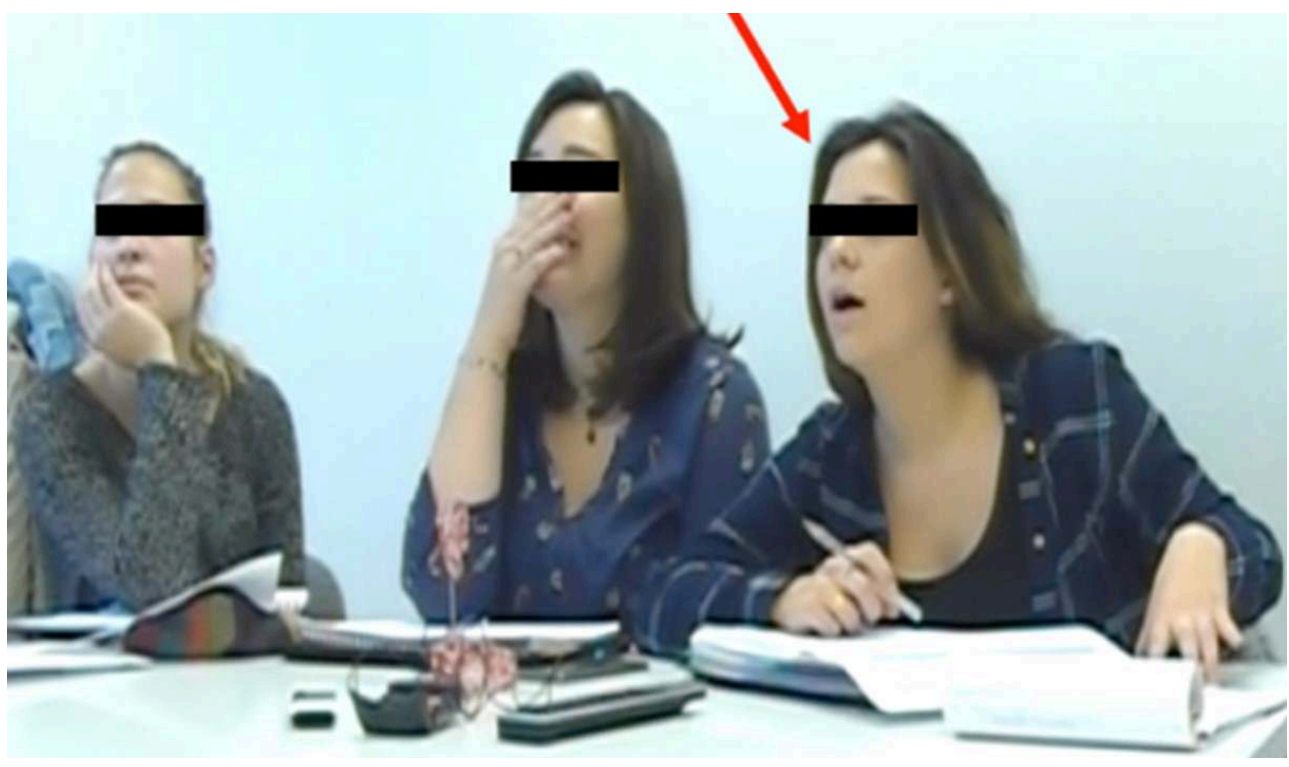

41 Elle ressent de l'empathie pour le foetus : «mince! Inquiétude là » (sentiment [U 2.1]), en identifiant une altération de son bien-être: «le bébé ne va pas bien depuis un certain temps, avant même que ça commence » (discours privé émergent [U 3*1]). Elle perçoit la situation comme instable pour le nouveau-né à naître et construit le problème posé par le scénario. Elle s'immerge en situation et pronostique l'activité qu'elle imagine devoir déployer en situation visionnée : «il est en souffrance [...] on va avoir une réanimation du nouveau-né » (action symbolique [U 3*2]) pour stabiliser la situation puis, dans un second temps, teste la fiabilité de son hypothèse (auto-réflexion 
[U 3*3]) (Annexe 1). Elle s'engage alors dans une activité d'enquête (Dewey, 1993) et adopte une posture distanciée, analytique, de la situation. Elle explore l'environnement simulé : «je regarde les quatre écrans », oriente son observation sur l'aménagement de l'espace dédié à la réanimation du nouveau-né (impulsion [U1.1]') et le compare avec les séances passées: "la table de réanimation, elle n'est pas comme d'habitude » (action [U 2.2]'). Elle s'étonne "qu'un champ dédié au séchage du nouveau-né soit déplié sur la table», et se dit qu'il va nécessairement être utilisé en cours de séance (discours privé émergent [U $\left.\left.3^{*} .1\right]^{\prime}\right)$. Elle partage les indices sélectionnés avec les autres observatrices présentes : "regarde la table est ouverte» (communication [U3*.2]'), rendant compte de la dimension coopérative de l'activité d'enquête. Elle fait dialoguer les éléments sélectionnés avec sa suggestion qui est alors envisagée comme la solution déterminant la situation-problème posé par les formateurs : «quand je vois ça, je me dis il va y avoir une réanimation" (auto-réflexion [U3*3]') (Annexe 2). La compréhension de la situation problème s'accompagne d'une poursuite de l'expérience mimétique initiale avec une projection empathique de soi dans l'autre, le fotus, mais aussi les actrices (sentiment [U 2.1]") en raison de l'identification de nouvelles indéterminations quant au devenir de l'enfant et quant à la pertinence des actions menées par ses paires en situation (discours privé émergent [U $\left.3^{*} .1\right]$ "). En effet, l'observation se déroule en direct, ce qui implique pour Estelle d'être témoin, sans pouvoir s'y soustraire, d'une situation de simulation sans montage, ni ellipse temporelle qui peut donner à voir de l'inattendu, de l'imprévisible (Jost, 2015) : « je ne suis pas confortable, je sais que ça ne va pas aller pour le bébé, j'appréhende de voir ce qui va se passer et comment elles vont gérer la situation ». Elle se prépare à vivre par procuration une situation inédite. Elle adopte une attitude ludique ${ }^{15}$ (Henriot, 1983) en considérant la situation dans laquelle elle s'engage comme comportant de l'incertitude.

\subsubsection{Empathie, engagement corporel et évaluation}

42 Le second moment choisi se situe lors du dégagement fœtal au cours d'une séance de simulation nommée « hémorragie de la délivrance » (Figure 13).

Figure 13 : Projection filmique de l'environnement simulé. Figure 13: Filmic projection of the simulated environment

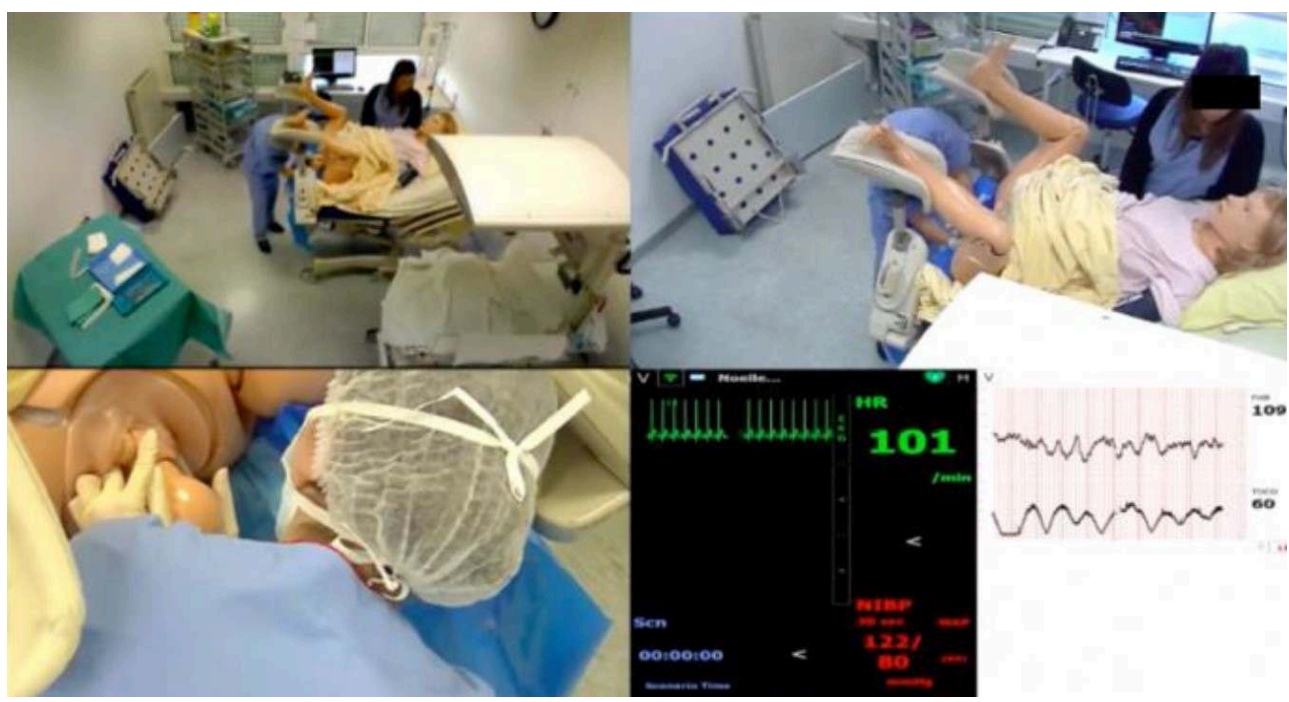



visant à découvrir la situation problème prévue par les formatrices. La parturition et l'expulsion étant évaluées jusqu'alors comme physiologiques, Laurène ne parvient pas à sélectionner dans la situation observée des indices prédictifs de la complication clinique attendue. Cette indétermination prolongée suscite du suspens: «il y a tellement d'attente que c'est horrible à dire, mais je veux vraiment qu'il y ait quelque chose là ». À l'instant considéré, elle regarde les manœuvres de dégagement fœal réalisées (action [U 2.2]) : «je regarde en bas à gauche ce qu'elle fait là, les gestes ». Elle constate que le fotus ne se dégage pas (action symbolique [U $\left.3^{*} 2\right]$ ) : «il est coincé le pauvre bichou ", et ressent de l'inconfort pour le fœetus (sentiment [U 2.1]), mais aussi pour l'actrice en situation : « elle galère avec le mannequin » (Annexe 3). L'immersion empathique l'amène à ressentir l'inconfort vécu par autrui, à adopter la perspective visuelle et spatiale de l'actrice, par une translocation du point de vue égocentré dans le corps de la personne observée et par la mesure des différences qui contribuent à ne pas s'identifier à elle. Laurène estime que la manœuvre de restitution n'est pas optimale (discours privé émergent [U $\left.3^{*} 1\right]$ ): «elle ne l'a pas trop restitué en fait». Afin d'accorder une signification aux évènements observés et vérifier son hypothèse, elle s'engage fictionnellement en situation devant la parturiente (tropisme [U 2.1]') : « là je suis devant le périnée ", se redresse et réalise la manœuvre précédemment observée (Figure 14), sans qu'il y ait de contact effectif avec le fotus (action [U2.2]') (Annexe 4).

Figure 14 : Extrait photographique de l'activité manifeste de Laurène (à gauche). Figure 14: Photographic extract of Laurène's observable activity

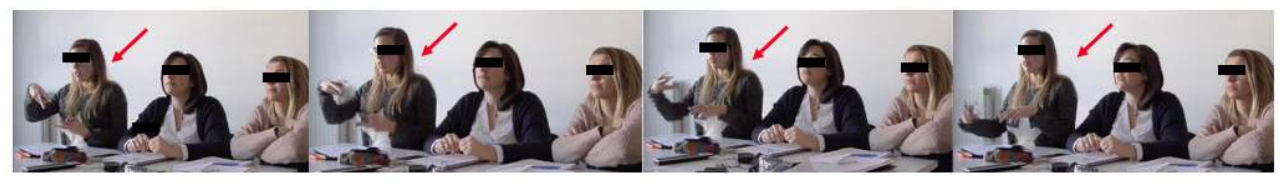

La réactivation des perceptions motrices qui accompagnent le geste, la simulation du mimème (Schaeffer, 1999) déjà réalisé lors de mises en situation antérieures, permet à Laurène de comparer la réalisation des deux manœuvres (action symbolique [U3*.2]'), et d'identifier une différence entre les activités. Elle confirme ainsi son hypothèse et celle des observatrices présentes (auto-réflexion [U3*3]') : « elle ne l'a pas bien mis dans l'axe, que c'est pour cela qu'il est bloqué » (Annexe 4). Le retard de dégagement foetal n'a pas pour étiologie une dystocie des épaules comme le propose une autre observatrice, mais est bien la conséquence d'un manque de dextérité de l'actrice en situation. L'indétermination de la complication prévue par les formatrices persiste et accompagne la poursuite de l'activité collégiale d'enquête.

L'application de l'élaboration théorique, à travers ces exemples, permet de documenter comment les processus mimétiques à l'œuvre, dans ce contexte de formation, ont pour ancrage un partage émotionnel avec les différents protagonistes de la situation observée, engageant les apprenantes à planifier des d'actions, à produire des gestes techniques fins de la pratique, tout en établissant des comparaisons entre les activités en cours et les expériences passées ${ }^{16}$. Ce jeu de comparaison s'accompagne d'une forte activité interprétative avec mobilisation de connaissances spécifiques à la pratique permettant d'évaluer la situation, de pronostiquer son évolution, et ainsi d'engager de nouvelles actions jusqu'à sa stabilisation. 


\subsection{Pister des transformations sur le temps long dans un espace de formation à la conduite de chevaux attelés}

Dans ce dernier cas (voir 3.3), nous nous intéressons à la situation de visionnage d'une stagiaire-meneur d'attelage. Les expériences mimétiques ne transforment pas seulement l'activité de visionnage des stagiaires, mais ont des effets sur leur activité de conduite. La recherche sur l'apprentissage/développement des meneurs illustre ces transformations sur le temps long (6 mois). Nous repérons dans son activité des expériences mimétiques de différents ordres. Ces expériences ont la caractéristique d'établir une tension entre ce que la stagiaire perçoit de son activité de meneur d'attelage et ce qu'elle perçoit de l'activité d'autres meneurs d'attelage. Les transformations vécues lors du visionnage ont des répercussions dans les jours qui suivent la séance de vidéoformation: discussion avec les formateurs, poursuite d'enquête, nouvelle sensation lors de la conduite.

\subsubsection{Comportement immersif et prise de conscience}

Naviguant librement sur la plateforme de vidéoformation, la stagiaire sélectionne une vidéo du thème " communication ». La présence des mots " contact » et "équilibré » dans le titre l'interpelle. Elle entend en filigrane la voix de son formateur comme un écho imaginaire : "Vincent me fait/ enfin me dit souvent: "et là, tu ré-équilibres ton cheval" et je comprends pas toujours». En lançant la vidéo, elle attend des réponses à cette question d'équilibre du cheval. Le début de la vidéo projette la stagiaire à bord d'un l'attelage sur une petite route de campagne. C'est agréable. Son corps se met en mouvement en se balançant (Tableau 1).

Tableau 1 : Verbatim de la stagiaire au sujet de son bercement corporel.

Tableau 1: Verbatim transcript of the trainee about her corporal rocking

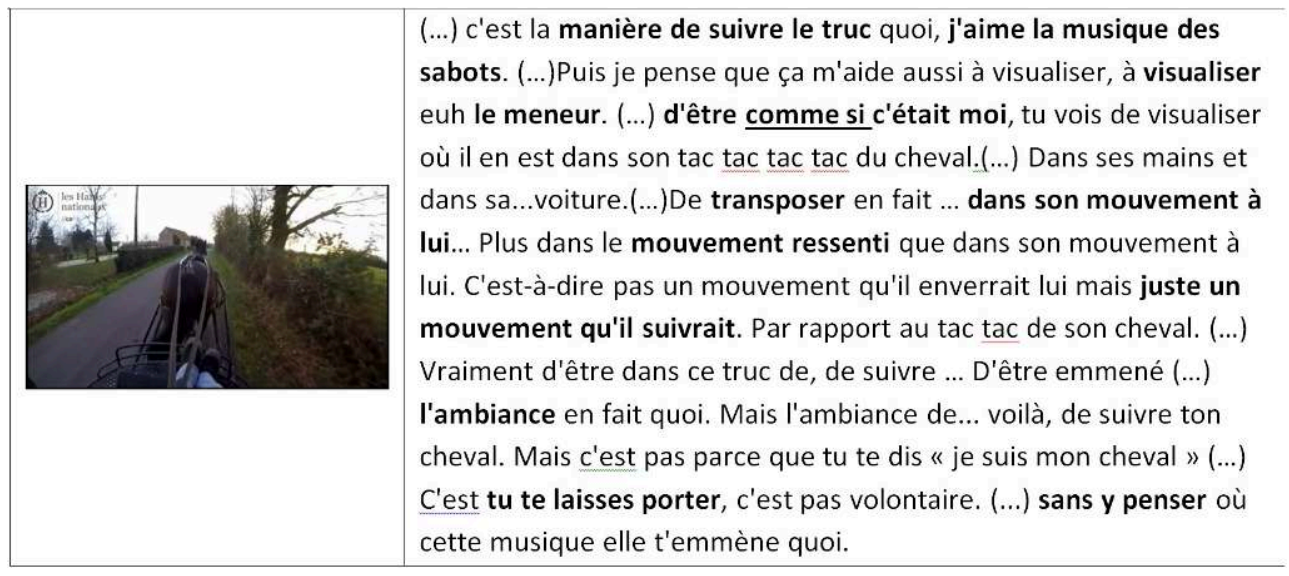

Le bruit des pas du cheval attelé est perçu comme une mélodie par la stagiaire. En dépit $\mathrm{du}$ fait qu'elle soit assise devant l'écran d'ordinateur, Nadège vit une immersion mimétique intense : elle traverse en imagination l'écran, se projetant involontairement dans le ressenti du corps du meneur de la vidéo. Cette projection n'est pas visuelle, elle est un mouvement intérieur. En revanche, sa réaction corporelle est visible à travers un balancement cadencé. 
L'analyse de ce moment permet de construire le signe suivant (Tableau 2). La stagiaire vit une impulsion [U.1.1] qui s'accompagne d'un sentiment agréable [U.2.1] et d'une réaction corporelle [U.2.2]. L'élément significatif qui fait partie de ce signe est un representamen de l'ordre de l'émergence d'une forme globale [R.2.1]. Il s'ancre dans un ensemble de qualités particulières : le visionnage d'un environnement et d'une musique agréables. Il sous-tend un representamen de l'ordre de l'apparition d'un fond [R.1.1]: une ambiance agréable.

Tableau 2: Hexatomie du U d'un signe.

Tableau 2: $U$ hexatomy of a contemplative/passion sign

Image

102FEBB00000455C00003794184DC7E44ABE255F.emf

\begin{tabular}{|c|c|c|c|c|c|}
\hline $\begin{array}{c}\text { Unité } \\
\text { élémentaire }\end{array}$ & $\begin{array}{c}\text { Represen- } \\
\text { tamen }\end{array}$ & Engagement & $\begin{array}{c}\text { Actualité } \\
\text { potentielle }\end{array}$ & Référentiel & Interprétant \\
\hline $\begin{array}{l}\text { [U.1.1] } \\
\text { Impulsion: } \\
\text { emmenée par } \\
\text { la musique des } \\
\text { sabots du } \\
\text { cheval } \\
\text { [U.1.2] } \\
\text { sentiments : } \\
\text { "J'adore le } \\
\text { bruit des } \\
\text { sabots" } \\
\text { curiosité } \\
\text { [U.2.2] } \\
\text { [ré]action: } \\
\text { suit la battue } \\
\text { du cheval en } \\
\text { balançant son } \\
\text { corps en } \\
\text { rythme }\end{array}$ & $\begin{array}{l}\text { [R.1.1] une } \\
\text { ambiance } \\
\text { agréable } \\
\text { [R.2.1] } \\
\text { émergence } \\
\text { d'une forme } \\
\text { globale: } \\
\text { musicalité du } \\
\text { bruit des } \\
\text { sabots «tac } \\
\text { tac, tac tac » }\end{array}$ & 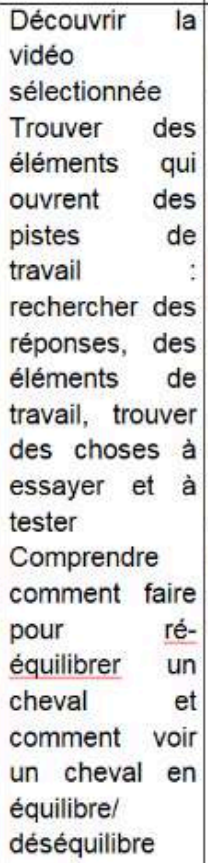 & $\begin{array}{l}\text { "vidéo Vers } \\
\text { un contact } \\
\text { équilibré " } \\
\text { étant en accord } \\
\text { avec son titre : } \\
\text { apportant } \\
\text { encore des } \\
\text { informations } \\
\text { visuelles } \\
\text { apportant une } \\
\text { explication } \\
\text { " je " } \\
\text { découvrant la } \\
\text { vidéo; retenant } \\
\text { des } \\
\text { informations } \\
\text { offertes par la } \\
\text { vidéo }\end{array}$ & $\begin{array}{l}\text { C'] ai beaucoup } \\
\text { de mal à tenir } \\
\text { un cheval en } \\
\text { équilibre; ne } \\
\text { comprend pas } \\
\text { quand re re } \\
\text { formateur me } \\
\text { dit de "ré- } \\
\text { équilibrer" mon } \\
\text { cheval } \\
\text { [formateur] me } \\
\text { dit souvent: " } \\
\text { Et là, tu ré- } \\
\text { équilibres ton } \\
\text { cheval" } \\
\text { [vidéos } 1 \text { \& } 2 \text { ] } \\
\text { sont } \\
\text { essentiellemen } \\
\text { t visuelles ; ont } \\
\text { un titre en } \\
\text { décalage avec } \\
\text { le contenu }\end{array}$ & $\begin{array}{l}\text { [1.2.1] } \\
\text { Renforcement } \\
\text { de type : } \\
\text { [musique des } \\
\text { sabots] } \\
\text { est agréable }\end{array}$ \\
\hline
\end{tabular}

50 Au moment où son corps se balance, la stagiaire n'a pas conscience de se balancer sur le rythme des pas du cheval. Elle n'y pense pas et le fait sans y prêter garde: "Mais l'ambiance de... voilà, de suivre ton cheval. Mais c'est pas parce que tu te dis "je suis mon cheval" ". De même, dans ce moment où la stagiaire se laisse envahir par la musicalité, les points d'appui de la conscience s'évanouissent et son engagement précédent (trouver des pistes de travail) s'estompe temporairement devant une forme d'écoute plaisir, sans intention particulière. Dans cet instant, l'écran d'ordinateur et la situation de visionnage $\mathrm{e}^{17}$ disparaissent momentanément. La stagiaire dit être «transposée » dans le «mouvement ressenti » du corps du meneur de la vidéo qu'il «suivrait par rapport au tac tac de son cheval». Dans cette perception-plaisir de la stagiaire qui entraine une expérience mimétique corporelle, le corps du meneur s'accorde/se synchronise à la battue du cheval. La nature de ce signe est d'ordre passionnel, tel qu'il peut se rencontrer dans l'activité de contemplation (Theureau, 2004). Ce plaisir, cette écoute, ce bercement sous la forme d'un laisser-aller sont difficilement actualisables en contexte de conduite réelle du fait de la dynamique et de l'intensité propres à l'activité, surtout chez des débutants. Dans la conduite d'un 
attelage, les engagements liés à la responsabilité, la maîtrise, la sécurité, l'application correcte du geste prennent le dessus sur les autres préoccupations dans le cours d'action. Dans ces situations, la musicalité des pas du cheval, l'écoute plaisir, le sentiment d'harmonie, sont absentes. En revanche, le contexte simulé offert par la vidéo permet l'exploration de ce vécu passionnel. L'intervention de la chercheuse sort la stagiaire de cette expérience mimétique de premier degré (expérience mimétique de $1^{\text {er }}$ degré = sans U symbolique), interrompant son cours d'action contemplatif.

51 La vidéo se déroule et la stagiaire continue de la regarder. Ce qui lui apparaît alors de manière significative, ce ne sont plus les pas du cheval et leur musicalité, mais l'absence de la voix du meneur. Elle décèle à cet instant un écart entre le contenu de la vidéo et le thème " communication ». Ses engagements relatifs à l'ouverture de pistes de recherche pour sa conduite reviennent au premier plan de son cours d'action. L'évolution de son action de visionnage est permise par la prise de recul entre l'immersion sensorielle mimétique et la prise de conscience d'une absence étrange: « on n'a encore pas entendu le son de sa voix » (Tableau 3).

Tableau 3 : Verbatim de la stagiaire relative à l'émergence d'une enquête.

Tableau 3: Verbatim transcript of the trainee in relation to the emergence of an inquiry

\begin{tabular}{|l|l|}
\hline 1.6. & $\begin{array}{l}\text { C'est marrant parce qu'on est dans la communication et on n'a encore pas } \\
\text { entendu le son de sa voix. Je suis surprise par la réflexion que nous a fait } \\
\text { Vincent vendredi dernier, [noir à l'écran] de nous dire qu'on ne parlait pas } \\
\text { assez à nos chevaux. [on entend le bruit des chevaux qui reprend avant } \\
\text { l'image] Du coup je cherche depuis [le passage d'autoconfrontation du } \\
\text { meneur commence] ce, ce rythme qu'il faudrait avoir dans le dialogue. }\end{array}$ \\
\hline
\end{tabular}

Le mutisme du meneur de la vidéo surprend la stagiaire, car, dans son cours de vie relatif à la formation à la conduite de chevaux attelés, elle est en train de s'approprier la notion de communication avec un cheval attelé. Cette communication passe principalement par l'action des mains du meneur et d'une façon complémentaire par l'intermédiaire de la voix et du fouet. Elle sait qu'elle a à y travailler pour agir de façon adéquate aux attendus culturels. Mettant à distance son vécu immersif contemplatif/ passionnel, la stagiaire relève un paradoxe entre le thème "communication » de la vidéo et le contenu "muet» de la vidéo. Le contraste entre l'annonce du thème " communication ", générant l'attente de l'usage de la voix par le meneur, et le silence de ce même meneur, crée chez Nadège une étrangeté ([U.2.1] tropisme), quelque chose d'inattendu ouvrant une intrigue (Annexe 5). Ce silence n'a pas tout de suite été perçu puisque la battue des pas du cheval avait envahi précédemment le couplage de la stagiaire avec la vidéo. À présent que ce meneur muet est révélé, cet inattendu fait émerger une expérience antérieurement vécue par la stagiaire en lien avec l'usage de la voix pour communiquer avec le cheval (J-4). L'expérience mimétique vécue ici d'une similitude de situations entre le meneur qui ne parle pas à son cheval et, eux, les stagiaires, dont le formateur dit qu'ils ne parlent pas suffisamment à leurs chevaux, réactive une enquête ouverte précédemment: comment établir la communication verbale suffisante avec son cheval? 


\subsubsection{L'expérience d'autrui transformant l'expérience du stagiaire visionnant}

53 La deuxième partie de la vidéo présente un entretien du meneur qui explique la conduite qui vient d'être visionnée par la stagiaire. Jusqu'à présent, le contenu de la vidéo jugé potentiellement utile par la stagiaire était principalement visuel et sonore. Cela avait pour effet de la plonger dans une activité d'observation-contemplative puis d'observation-recherche (d'indices visuels et sonores). Le changement de contenu, s'orientant sur les commentaires du meneur, place la stagiaire dans une activité d'écoute-compréhension à la recherche de nouveaux indices, cette fois-ci, discursifs. Nous observons dans cette activité d'écoute de nouvelles formes d'expériences mimétiques. L'interaction de la stagiaire avec les commentaires du meneur de la vidéo produit différents effets: questionnement ouvert envers un meneur absent, reconnaissance d'un problème partagé avec le meneur de la vidéo, répétition en écho des mots du meneur de la vidéo retenant l'attention de la stagiaire, rejeu ciblé du début de la vidéo (permettant de voir l'invisible du premier visionnage) (Tableau 4).

Tableau 4 : Interactions de la stagiaire dans la situation de visionnage médiée par la chercheure. Tableau 4: Trainee's interactions in the viewing situation mediated by the researcher

\begin{tabular}{|c|c|c|c|c|c|}
\hline $\begin{array}{l}\text { Meneur de la } \\
\text { vidéo }\end{array}$ & $\begin{array}{l}\text { je vais vraiment } \\
\text { mettre du poids. } \\
\text { Et puis quand il } \\
\text { va me lâcher, je } \\
\text { dirai, la main, à } \\
\text { ce moment-là, j'ai } \\
\text { ma main qui va } \\
\text { avancer }\end{array}$ & $\begin{array}{l}\text { j'essaye de } \\
\text { l'avoir droit. } \\
\text { Là, on voit } \\
\text { bien qu'il se } \\
\text { déplace } \\
\text { légèrement } \\
\text { vers la } \\
\text { gauche }\end{array}$ & $\begin{array}{l}\text { ce coup-ci, je } \\
\text { vais vraiment } \\
\text { reculer ma } \\
\text { main droite, } \\
\text { on le voit } \\
\text { bien tu vois } \\
\text { puisqu'elle } \\
\text { disparait un } \\
\text { peu à l'image }\end{array}$ & $\begin{array}{l}\text { (le meneur } \\
\text { poursuit ses } \\
\text { commentaires) }\end{array}$ & $\begin{array}{l}\text { Là on voit bien } \\
\text { mes mains qui } \\
\text { se reculent qui } \\
\text { ravancent voilà } \\
\text { et je suis } \\
\text { vraiment en } \\
\text { train de } \\
\text { discuter avec } \\
\text { lui (...) }\end{array}$ \\
\hline Effet & Questionnement & Partage & $\begin{array}{l}\text { Re-jeu } \\
\text { demandé }\end{array}$ & Re-jeu différé & $\begin{array}{l}\text { Répétition en } \\
\text { écho }\end{array}$ \\
\hline $\begin{array}{l}\text { Stagiaire } \\
\text { visionnant }\end{array}$ & $\begin{array}{l}\text { Donc tu mets du } \\
\text { poids en ti/ en } \\
\text { reculant ta } \\
\text { main? }\end{array}$ & $\begin{array}{l}\text { Ouais ça c'est } \\
\text { mon problème } \\
\text { (en } \\
\text { chuchotant) }\end{array}$ & $\begin{array}{l}\text { On peut } \\
\text { revoir nous } \\
\text { ça aussi? }\end{array}$ & $\begin{array}{l}\text { Je re- } \\
\text { regarderai } \\
\text { après quand il } \\
\text { aura fini de } \\
\text { parler. }\end{array}$ & $\begin{array}{l}\text { "j'suis en train } \\
\text { de } \\
\text { discuter avec } \\
\text { lui " } \\
\text { "y a pas de } \\
\text { moment où je } \\
\text { fais rien" }\end{array}$ \\
\hline
\end{tabular}

Suite à cette vidéo, la stagiaire rejoue la vidéo: «Fais voir, je veux re-regarder maintenant ! » Elle est à la recherche visuelle du déséquilibre du cheval dont a parlé le meneur : «C'est de l'excitation, parce que je sais que je vais voir plus de choses! » Elle perçoit différemment l'activité de conduite du meneur, éclairée par les commentaires, récits, monstrations de celui-ci : «Ah oui c'est là. Tu vois c'est là où le cheval il a tourné/ tu vois comme il tourne un petit peu sa tête. Et où, lui, du coup, il rappelle à droite." Cette séquence s'accompagne de deux principales transformations de connaissances-types présentes chez la stagiaire: la notion de poids et la notion de discussion avec les mains. Elle découvre une nouvelle façon de parler du contact : en termes de poids et pas seulement de tension. Elle apprend qu'en reculant la main, le meneur met du contact dans la guide. C'est l'association de son engagement et de perceptions dans le discours du meneur de la vidéo validée par un nouveau visionnage éclairé qui permet la transformation du référentiel de la stagiaire. Cet apprentissage est majeur dans son cours de vie: il va réorienter ses préoccupations, perceptions et recherches d'actions en conduite attelée.

"Quand je l'entends dire "j'suis en train de discuter avec lui" alors qu'il a pas

échangé une seule parole avec son cheval, que pour lui "discuter" c'est dans ses 
mains, tu vois, au niveau du contact ça t'ouvre des perspectives plutôt que de voir tes mains juste pour dire/ parce que finalement, tes mains, t'as l'impression au bout d'un moment dans l'attelage que t'es là pour contraindre quand même pour, pour dire ce qu'il faut faire en permanence. Ce qui est vrai. Mais quand tu discutes, t'as pas cette impression d'ordonner. T'as vraiment cette impression du coup de chercher le contact et d'être en accord avec ton cheval »

\subsubsection{Des prolongements en dehors de l'expérience de vidéoformation : pistes de recherche (réflexives, corporelles et perceptives), échanges avec formateurs} lendemain du visionnage lors d'une séance de conduite avec un formateur. Il s'agit de deux histoires laissées en suspens lors des visionnages. C'est-à-dire qu'elles ont ouvert des pistes de transformation de sa pratique, pour lesquelles la stagiaire a besoin d'avoir confirmation par le formateur. En sollicitant ses formateurs, elle s'assure d'être en conformité avec les attendus de la formation. L'histoire du fouet émerge pendant qu'elle est train de conduire. Le formateur vient confirmer son idée de « pousser avec le fouet » (Tableau 5). Nous supposons que la stagiaire valide cette histoire comme type d'action valide du fouet.

Tableau 5 : L'histoire du fouet ré-émergeant le lendemain du visionnage. Tableau 5: The story of the whip re-emerging the day after the viewing

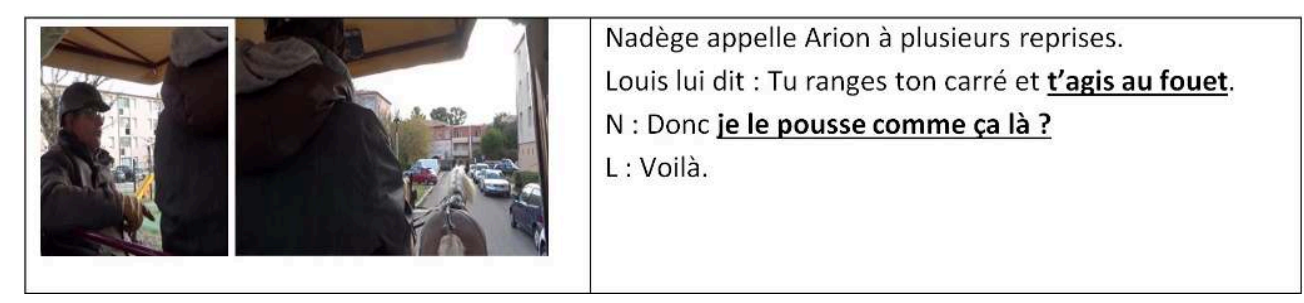

57 L'histoire du poids, elle, a donné lieu à une discussion avec le formateur après sa conduite. Elle entend les nuances apportées par le formateur, cependant cette interaction ne donne pas entière satisfaction à la stagiaire et laisse l'histoire du poids en suspens. Elle projette de poursuivre l'enquête sur cette histoire du poids qui l'accompagnera jusqu'à la fin de la formation. Le surlendemain, pendant sa conduite, une transformation significative émerge. Elle prête attention pour la première fois à un ressenti léger dans les guides, alors que jusqu'à présent c'était le lourd qui attirait son attention. Elle se rend compte qu'elle peut mener ses chevaux sans forcer et en légèreté. Cet élément est un apprentissage crucial dans le parcours du meneur d'attelage. Les expériences mimétiques vécues lors des visionnages vidéos d'autres meneurs ont mobilisé l'ensemble de l'expérience de la stagiaire par des imaginations sensorielles diverses : visuelles, auditives et somatiques. Ces expériences ont ensuite pu se prolonger dans d'autres contextes : en situation de conduite ou lors de discussion avec les formateurs. Nos résultats (Secheppet, 2020) montrent l'importance de la complémentarité des expériences sensibles et verbalisées dans les processus d'appropriation, ainsi que la nécessité d'allers-retours entre des expériences de pratique et des expériences de distanciation permises par l'interaction avec l'activité 
d'autres meneurs, l'interaction avec les formateurs, ou l'interaction avec d'autres stagiaires.

\section{Discussion}

\subsection{Expérience mimétique, relation iconique et mouvement (jeu)}

Dans l'objectif de comprendre, décrire et expliquer l'expérience mimétique dans des situations de vidéoformation, nous questionnons une relation particulière au sein du couplage acteur-environnement: le rapport d'expérience soi/autrui constitué dans l'activité mimétique (Wulf, 2007). Ce rapport inclut une bivalence "similitude / différence " caractéristique de l'activité de comparaison (Horcik \& Durand, 2015; Poizat \& Goudeaux, 2014). Cette bivalence ouvre des liaisons inédites porteuses de déstabilisation et de développement pour les formés. Le rapport de proximité/distance entre soi et autrui ne se limite toutefois pas à une activité de type réflexif. Au contraire, les manifestations premières de ce rapport sont souvent vagues, floues. Elles correspondent à des impressions, des intuitions, des sensations, des attirances ou des répulsions. Le point commun de ces manifestations premières est leur caractère faiblement déterminé. La documentation de l'hexatomie du U nous permet de mettre en évidence que l'expérience mimétique se développe à partir d'une forme peu déterminée ([U.1.1] et [U.2.1]) et chemine vers une forme mieux déterminée, accompagnée de symbolique ([U.3*.2] et [U.3*.3]). Les unités d'expérience indéterminées sont de genre dégénéré ${ }^{18}$ et se révèlent dans un rapport au monde des sens, sur le mode d'une relation floue caractéristique de l'icône : «[...] L'icône n'indique pas comme le fait l'indice, elle suggère, elle laisse entendre ou apercevoir, mais comme dans un flou ou dans une incertitude" (Fisette, 2012, p. 278). Cette relation s'accompagne d'un faible degré de certitude et de stabilité. Les caractéristiques sensitives et peu déterminées de la relation iconique expliquent le fait que ces expériences sous-jacentes soient difficiles à documenter. Nous retrouvons ce caractère de faible détermination dans les travaux sur la métaphore menés par Durand, Goudeaux, Horcik, Salini, Danielan et Flobert (2013) : «C'est là un registre où les significations sont peu déterminées, où les choses valent pour d'autres choses de façon équivoque, désintéressée, oscillante, plaisante, erratique. Elles sont porteuses de concentrations, dédoublements, décalages, détournements, décentrations... par rapport à un engagement finalisé et univoque " (p.55). Nous rejoignons leurs observations : "La fécondité de la métaphore pour l'apprentissage tient à ce que sa signification établit une ressemblance nouvelle par une mise en relation absente au préalable " (Ibid., p. 58). Mais avant d'être nette, cette relation est chaotique. Nous prolongeons la piste de la relation iconique ouverte par ces auteurs, par l'intérêt porté à l'hexatomie du U et ses composantes faiblement déterminées. Fisette (1998/1999) avance l'idée que l'incertitude laissée par l'icône rend possible un "assouplissement de la référence » et donc une ouverture et une mise en mouvement : cela crée un espace de jeu. Cet espace de jeu est un espace d'action potentielle qui permet le déplacement. Ce déplacement peut être un retour en arrière (forme de régression) avant de provoquer une extension de l'activité. «Ce que la figure de l'icône perd en valeur de vérifiabilité, elle le gagne en pouvoir de suggestion; en somme, il y a une perte de compréhension, mais pour un gain d'extension" (Fisette, 2012, p. 278). Ainsi l'icône apparaît comme un espace de liberté pour l'imaginaire qui, à la fois, fragilise et enrichit le signe. Poizat, Salini et 
Durand (2013) évoquent une «modalité iconique de signification » (p. 105) propice aux rapports entre expériences mimétiques et apprentissage. En outre, Fisette (2012) souligne que l'icône fait appel à tous les sens : visuel, sonore, olfactif, tactile, gustatif. Gal-Petitfaux (2015) a mis en avant l'importance de cette immersion sensorielle dans le cadre de la formation des enseignants: "Ces instruments [la vidéoformation] ont facilité une approche pragmatique et incarnée des situations de classe : les étudiants ont identifié non pas des idées ou des concepts en premier, mais des mondes vivants (avec des évènements et des activités de travail) et des mondes vécus (évocation de souvenirs professionnels)»(p.16). Du fait que l'expérience mimétique se caractérise par une mise en relation entre sa propre activité et celle d'autrui, elle est un registre d'expérience toujours singulier au sein duquel l'engagement des acteurs aux situations est complexe. Il n'existe pas d'expérience mimétique sans cette relation soi-autrui qui est porteuse « de transformations de l'activité en raison des décalages, concentrations, dédoublements, détournements, décentrations, etc., qu'elle génère par rapport à un engagement finalisé et univoque » (Ibid., p. 41). Les environnements de vidéoformation et/ou de simulation proposent des situations emblématiques faisant apparaître cette expérience mimétique.

\subsection{Intérêts et limites de ces dispositifs de formation à fort potentiel immersif}

59 La conception des trois dispositifs de formation présentés a pour point commun de créer des environnements au fort potentiel immersif, grâce à l'usage d'artefacts vidéo qui constituent des offres ou des possibles d'actions simulées, imaginées pour les formés. Les médiations filmiques se présentent comme des vecteurs d'immersion (Schaeffer, 1999) permettant d'une part de transformer l'environnement des formés, en leur offrant l'opportunité de partager le monde des acteurs observés, et d'autre part de perturber leur activité. Les amorces mimétiques (circonscription d'une zone situationnelle de performance [U.1.1]) sont inhérentes au statut sémiotique des flux imagés visionnés en direct ou en différé (Ibid.) et au degré de familiarité que le formé entretient avec la situation visionnée (Flandin, Leblanc, Muller, Roche, Blanes Maestre, Gal-Peitfaux et al., 2018). La focalisation sur des aspects de la pratique est soit à l'initiative de l'apprenant qui navigue sur une plateforme et sélectionne, selon ses préoccupations actuelles, des situations ordinaires, mais peu maitrisées (activité de conduite de chevaux attelés), voire critiques (difficultés en début de cours), soit imposée par un visionnage en direct de situations extraordinaires, à l'issue incertaine (prise en charge d'un travail marqué par une asphyxie fœtal per natale). Quel que soit le contexte, l'objectif est similaire: contribuer à un apprentissage-développement se traduisant par une amélioration des performances lorsque les formés seront engagés dans des situations réelles de travail, jugées similaires. Ainsi, ces dispositifs s'inscrivent: a) dans une approche développementale, c'est-à-dire qu'ils visent à encourager l'émergence de questionnements sources d'acquisition « de modes d'action nouveaux issus du patrimoine culturel professionnel » (Durand, 2009, p. 98), et b) dans une approche proscriptive (tout ce qui n'est pas proscrit est autorisé) s'éloignant d'une approche normative basée sur la monstration de l'activité d'un expert, qui viserait « ce qu'il faut faire» en vue d'une reproductibilité (Leblanc, 2014). Selon nous, une approche normée laisserait peu d'espace à l'adoption de postures mimétiques consistant à s'immerger dans le monde des observés (Tropisme [U2.1]), et à l'expression 
d'expériences mimétiques et fictionnelles singulières, créatives, et supports d'apprentissages professionnels (Wulf, 2007). Selon Wulf (Ibid.), «les processus mimétiques ne sont pas de simples processus d'imitation, de reproduction ou d'empreinte. Ils impliquent au contraire des mises en figure singulières, qui laissent place aux conditions et aux différences individuelles» (p. 23). Ainsi, deux vecteurs d'immersion enchâssés ont pu être identifiés, révélant une oscillation constante de proximité et de distance des formés avec les observés. D'une part, une position d'observation (Schaeffer, 1999) traduisant une immersion perceptive impersonnelle « je vois et entends ce que voit et entend l'observé ", «je recrée le monde de l'observé » (Action [U.2.2]), et d'autre part une substitution d'identité (Ibid.) traduisant un vécu par procuration "je ressens (Sentiment [U.2.1]) et je fais comme si je suis l'observé " (Action symbolique [U.3.*2]). La position d'observation se prolonge en activité d'analyse (Idéation [U.3.*1]) et d'évaluation de la situation visionnée ainsi qu'une mise en relation de l'activité imaginée à y déployer avec celle des observés (Auto-réflexion [U.3.*3]). La substitution d'identité physique «implique à la fois une identification personnifiante et une mimésis actancielle» (Schaeffer, 1999, p. 253). Cette identification allo-subjective actancielle se manifeste par des réactions émotionnelles à valence positive ou négative (Sentiment [U.2.1]), et intentionnelles, manifestées par l'exécution fictive d'actions (Déplacements, gestes imaginés ou réalisés), sans toutefois avoir d'incidence effective sur les environnements dans lesquels s'immergent les formés (Action symbolique [U.3*.2]). Ces deux postures sont à encourager, elles participent à l'instauration d'une réflexion professionnelle par confrontation de sa propre pratique avec celle d'autrui, à l'identification de différences réelles ou de possibles inter ou intra-activités (Duboscq, \& Clot, 2010 ; Muller \& Lussi Borer, 2018) (Auto-réflexions [U.3.*3]), in fine à la production de différences que les actions soient imaginées ou effectives (Wulf, 2007).

Ainsi, ces dispositifs présentent plusieurs intérêts éducatifs relatifs aux expériences mimétiques et fictionnelles: a) découvrir et/ou expérimenter des situations professionnelles complexes ou exceptionnelles, b) «faire-émerger» sur la base des différentes expériences vécues des questions dont la pertinence est dictée par les significations construites par l'acteur lui-même, c) appréhender et comprendre différemment la complexité d'une situation de travail, d) euphémiser les conséquences des actions permettant de se libérer de la pression de tout risque pour soi comme pour un tiers, e) (ré)activer des états émotionnels, des sensations, et f) à s'engager sur du temps long ${ }^{19}$ dans l'élaboration d'histoires qui constituent un puissant levier d'apprentissage-développement.

61 Toutefois, en tant que concepteur de formation, il est prudent de considérer plusieurs points de vigilance pouvant impacter la portée transformative de tels environnements de vidéoformation. D'une part, la capacité d'observer et d'analyser la pluridimensionnalité d'une situation professionnelle ne va pas de soi. L'observation peut s'apparenter à une activité de butinage (Flandin et al., 2015), consistant à voir, regarder, écouter (Action [U 2.2]). Dans ce cas, l'activité ne se prolonge pas en action symbolique ([U $\left.3^{*} .2\right]$ ) ni en auto-réflexion ([U $\left.3^{*} .3\right]$ ), ce qui en limite la portée formative. D'autre part, une séquence visionnée de façon solitaire peut soulever des questionnements sans réponses (idéation [U 3.*1]), ou encore l'observation collective peut s'accompagner de constructions collégiales erronées. Enfin, les états émotionnels (Sentiment [U 2.1]) qui émergent des expériences mimétiques et fictionnelles peuvent 
être de nature compassionnelle et conduire à un blocage, une immobilisation dans la conduite de l'activité (Leblanc \& Blanes-Maestre, 2018). Si ces expériences participent à des prises de conscience de sa propre gestion émotionnelle, par exemple l'absence d'empathie (Berthoz, 2003), elles peuvent impacter négativement le sentiment d'efficacité personnelle, donc conduire à une inhibition de l'action dans des situations similaires ultérieures (Bandura, 2007). Plusieurs leviers peuvent être mobilisés pour remédier à ces limites. Tout d'abord, il est possible d'instaurer un débriefing pour accompagner les processus de typicalisation (remédiation, renforcement) et pour assurer le passage de la spécificité de la situation observée-vécue à une plus grande généricité (transférabilité), en encourageant une posture d'auto et d'allo-analyse ainsi que le débat en cas d'observation collective (Bouchot, 2019). Ensuite, lorsque les équipements techniques le permettent, le redoublement des expériences de visionnage d'un même film contribuerait à provoquer (ou non) de nouvelles perturbations et à accompagner de nouvelles transformations ${ }^{20}$ (ou des renforcements). Enfin, se donner les moyens d'accéder aux préoccupations des formés, de repérer et suivre les enquêtes et les histoires qu'ils élaborent sur des temps plus ou moins longs dans ces espaces immersifs, et en dehors de ces espaces immersifs, doit permettre d'anticiper des scénarios de formation et d'aider les personnes à "connecter leurs expériences » (Saury, Adé, Gal-Petitfaux Huet, Sève, \& Trohel, 2013).

\section{BIBLIOGRAPHIE}

Azéma, G. (2015). L'improvisation selon les enseignants entrant dans le métier. Une approche en anthropologie cognitive. Thèse de doctorat non publiée, Université Montpellier 3.

Azéma, G., Secheppet, M., \& Mottaz, A-M. (2020). Envisager une ethnographie énactive? Réflexions illustrées. Activités (sous presse).

Bandura, A. (2007). Auto-efficacité: Le sentiment d'efficacité personnelle. Louvain-la-Neuve, Belgique : De Boeck.

Barbier, J-M. (2009). Voies pour la recherche en formation. Éducation et didactique, 3(3), 120-129.

Berthoz, A. (2003). La décision. Paris, France : Odile Jacob.

Bonnemain, A., Perrot, E., \& Kostulski, K. (2015). Le processus d'observation, son développement et ses effets dans la méthode des autoconfrontations croisées en clinique de l'activité. Activités, 12(2), 98-124. http://www.activites.org/v12n2/v12n2.pdf

Bouchot, H. (2019). Observer autrui lors de séances de simulation haute-fidélité: Une activité mimétique et ludique portée par en en-jeux sérieux. Thèse de doctorat en Sciences de l'Éducation et de la Formation, Université Montpellier.

Bouchot, H., \& Leblanc, S. (2019). Observer ses pairs lors de séances de simulation haute-fidélité : une activité de nature ludique portée par des en-jeux sérieux. Activités, 16(2). http:// journals.openedition.org/activites/4425 
Cahour, B. (2006). Les affects en situation d'interaction coopérative : proposition méthodologique. Le Travail Humain, 69(4), 379-400. doi : 10.3917/th.694.0379.

Dewey, J. (1993). Logique : La théorie de l'enquête (G. Deledalle, Trad.). Paris : PUF.

Duboscq, J., \& Clot, Y. (2010). L'autoconfrontation croisée comme instrument d'action au travers du dialogue : objets, adresses et gestes renouvelés. Revue d'anthropologie des connaissances, 4(2), 255-286. doi : 10.3917/rac.010.0255.

Durand, M. (2008). Un programme de recherche technologique en formation des adultes : une approche enactive de l'activité humaine et l'accompagnement de son apprentissage/ développement. Éducation \& Didactique, 3(2), 69-93.

Durand, M. (2009). La conception d'environnement de formation sous le postulat de l'enaction. In M. Durand \& L. Filliettaz (Eds.), Travail et formation des adultes (pp. 191-225). Paris, France: PUF.

Durand, M. (2013). Construction of dispositions and development of human activity. A theorical framework illustrated by the case of a novice manager. International Journal of Lifelong Education, 32(1), 39-55.

Durand, M., Goudeaux, A., Horcik, Z., Salini, D., Danielan, J., \& Frobert, L. (2013). Expérience, Mimesis et apprentissage. In L. Albarello, J.-M. Barbier, E. Bourgeois \& M. Durand (Eds), Expérience, activité, apprentissage (p. 39-64). Paris : PUF. http://www.cairn.info/experienceactiviteapprentissage--9782130619758-page-39.htm

Fisette, J. (1998/1999). Parler du virtuel. La musique comme cas exemplaire de l'icône. Protée, 26(3), 45-54. https://constellation.uqac.ca/2367/1/Vol_26_no_3.pdf

Fisette, J. (2012). Courte lecture de la notion d'icône chez Peirce. Intellectica, 58(2), 277-284. https://intellectica.org/fr/courte-lecture-de-la-notion-d-icone-chez-peirce

Flandin, S., Leblanc, S., \& Muller, A. (2015). Vidéoformation « orientée activité » : Quelles utilisations pour quels effets sur les enseignants. Analyse du travail et formation dans les métiers de l'éducation. Raisons éducatives, 19, 179-198.

Flandin, S., Leblanc, S., Muller, A., Roche, A., Blanes Maestre, C., Gal-Petitfaux, N., Lussi Borer, V., \& Ria, L. (2018). Une approche sémiologique de l'activité et de la vidéo-formation : Repères théoriques. In C. Gaudin, S. Moussay, S. Flandin, \& S. Chalies (Eds.), Vidéo-formation et développement de l'activité professionnelle enseignante (pp. 33-48). Paris : L'Harmattan.

Gal-Petitfaux, N. (2015). Faire de l'expérience vidéoscopée un moyen de formation au métier d'enseignant : l'appropriation d'un dispositif multimédia par des étudiants en éducation physique. Questions vives [en ligne], 24. http://journals.openedition.org/questionsvives/1854

Gaudin, C., Flandin, S., Moussay, S., \& Chaliès, S. (2018). Vidéo-formation et développement de l'activité professionnelle enseignante. Paris : L'Harmattan.

Henriot, J. (1983). Le jeu. Synonyme-S.O.R.

Horcik, Z., \& Durand, M. (2015). L'expérience mimétique dans l'apprentissage adulte : le cas des formations par simulation. Schweizerische Zeitschrift für Bildungswissenschaften, 37(1), 167-186.

Jost, F. (2015). La Télé-réalité: Grandeur et misère de la télé-réalité. Le Cavalier Bleu.

Kleiber, G. (1991). Prototype et prototypes : encore une affaire de famille. In D. Dubois (Ed.), Sémantique et cognition (pp. 103-129). Paris : CNRS-Editions. https://doi.org/10.4000/ rechercheformation. 
Leblanc, S. (2014). Vidéo formation et transformations de l'activité professionnelle. Activités, 11(2). https://doi.org/10.4000/activites.968

Leblanc, S. (2015). Expériences mimétiques en Vidéo formation et transformation de l'activité professionnelle. Recherche et Formation, 75, 37-50.

Leblanc, S. (2018). Analysis of Video-Based Training Approaches and Professional Development. Contemporary Issues in Technology and Teacher Education, 18(1), 125-148.

Leblanc, S., \& Blanes-Maestre, C. (2018). Immersion, réflexion, imagination et transformation via le visionnement de vidéos d'enseignants. In C. Gaudin, S. Moussay, S. Flandin, \& S. Chalies (Eds.), Vidéoformation et développement de l'activité professionnelle enseignante (pp. 65-94). Paris :

L'Harmattan.

Leblanc, S., Ria, L., Dieumegard, G., Serres, G., \& Durand, M. (2008). Concevoir des dispositifs de formation professionnelle des enseignants à partir de l'analyse de l'activité dans une approche enactive. Activités, 5(1), 58-78.

Leblanc, S., \& Sève, C. (2012). Vidéo formation et construction de l'expérience professionnelle. Recherche et Formation, 70, 47-60.

Maturana, H. R., \& Varela, F. J. (1994). L'arbre de la connaissance. Paris: Addison-Wesley.

Mollo, V. (2004). Auto- and Allo-confrontation as tools for reflective activities. Applied Ergonomics, 35 (6), 531-540.

Muller, A., \& Lussi Borer, V. (2018). Analyse de l'activité, environnement de vidéo-formation et développement professionnel des enseignants. Différences intra et inter-activités : Le possible, le réel et le virtuel. In C. Gaudin, S. Flandin, \& S. Moussay (Eds.), Vidéo-formation et développement de l'activité professionnelle enseignante (pp. 95-116). Paris : L'Harmattan.

Pastré, P. (2005). Apprendre par la simulation. De l'analyse du travail aux apprentissages professionnels. Toulouse: Octarès.

Petitmengin, C. (2006). Describing one's subjective experience in the second person. An interview method for the science of consciousness. Phenomenology and the cognitive sciences, 5, 229-269.

Poizat, G., \& Goudeaux, A. (2014). Appropriation et individuation : un nouveau modèle pour penser l'éducation et la formation? TF-Refa, 12, 13-38. https://transformations.univ-lille.fr/ index.php/TF/issue/view/1 - https://riviste.unimc.it/index.php/es_s/article/view/708/488

Poizat, G., Salini, D. \& Durand, M. (2013). Approche énactive de l'activité humaine, simplexité et conception de formations professionnelles. Éducation, Sciences \& Society, 4(1), 97-112. https:// riviste.unimc.it/index.php/es_s/article/view/708/488

Poizat, G., \& San Martin, J. (2020). Le programme de recherche « cours d'action » : repères historiques et conceptuels. Activités (sous presse).

Ria, L. (Ed.) (2010). Plateforme de formation en ligne Néopass@ction de l'Institution Français de l'Éducation de l'ENS de Lyon. http://neo.ens-lyon.fr/neopass

Ria, L., \& Leblanc, S. (2011). Conception de la plateforme de formation Néopass@ction à partir d'un observatoire de l'activité des enseignants débutants : Enjeux et processus. Activités, 8(2). https://doi.org/10.4000/activites.2618

Roche, L. \& Gal-Petitfaux, N. (2017). L'usage de la vidéo dans la formation des enseignants d'EPS : nouvelles perspectives d'usage. $7^{\text {ème }}$ Biennale internationale de l'AFRAPS, Pratiques sportives, éducation physique et société numérique : de l'outil à la transformation des rapports au corps, aux autres, au temps et à l'espace, AFRAPS, novembre, Mérignac, France. 
Rosch, E. (1973). Natural categories. Cognitive Psychology, 7, 328-350.

Saint Aubert (de), E. (2010). Espace et schéma corporel dans la philosophie de la chair de MerleauPonty. In A. Berthoz \& B. Andrieu (Eds.), Le Corps en Acte (pp. 125-152). Nancy : Presses Universitaires de Nancy.

San Martin, J. (2015). La culture d'action des enseignants de l'école primaire au Chili : contribution au développement d'une anthropologie énactive. Thèse de doctorat non publié, Toulouse, Université Toulouse II-Le Mirail.

Saury, J., Adé, D., Gal-petitfaux, N., Huet, B., Sève, C. \& Trohel,J. (2013). Actions, significations et apprentissages en EPS. Une approche centrée sur les cours d'expérience des élèves et des enseignants. Paris : EP\&S.

Schaeffer, J.-M. (1999). Pourquoi la fiction? Paris : Seuil.

Schaeffer, J.-M. (2002). De l'imagination à la fiction. Vox-poetica. Consulté le 23 février 2020. http://www.vox-poetica.org/t/articles/schaeffer.html

Secheppet, M. (2020). Apprendre dans un environnement de formation réel et simulé: Articulations d'expérience dans l'activité des cochers-meneurs d'attelage. Thèse de Doctorat, Montpellier, Université de Montpellier.

Sherin, M.G., Russ, R.S., Sherin, B.L., \& Colestock, A. (2008). Professional Vision in Action: An Exploratory Study. Issues in Teacher Education, 17(2), 27-46.

Terré, N., Sève, C. \& Saury, J. (2016). Une approche enactive du développement des compétences en milieu scolaire. Éducation et francophonie, XLIV(2), 68-85.

Theureau, J. (2000). Anthropologie cognitive \& analyse des compétences. In J. M. Barbier (Ed.), L'analyse de la singularité de l'action (pp. 171-211). Paris : PUF.

Theureau, J. (2004). Le cours d'action: Méthode élémentaire. Toulouse : Octarès.

Theureau, J. (2006). Le cours d'action: Méthode développée. Toulouse : Octarès.

Theureau, J. (2009). Le cours d'action: Méthode réfléchie. Toulouse : Octarès.

Theureau J. (2011). L'observatoire des cours d'action, des cours de vie relatifs à une pratique et de leurs articulations collectives. In G. Le Meur \& M. Hatano (Eds.), Approches pour l'analyse des activités (pp. 23-76). Paris : L'Harmattan.

Theureau, J. (2015). Le cours d'action : L'enaction \& l'expérience. Toulouse : Octarès.

Varela, F. J. (1989). Autonomie et connaissance-essai sur le vivant. Paris : Seuil.

Wulf, C. (2007). Une anthropologie historique et culturelle rituels, mimésis sociale et performativité. Paris: Téraèdre.

\section{ANNEXES}


Annexe 1: Sidérée devant le rythme fœtal.

Appendix 1: Astounded when confronted with the foetal rhythm

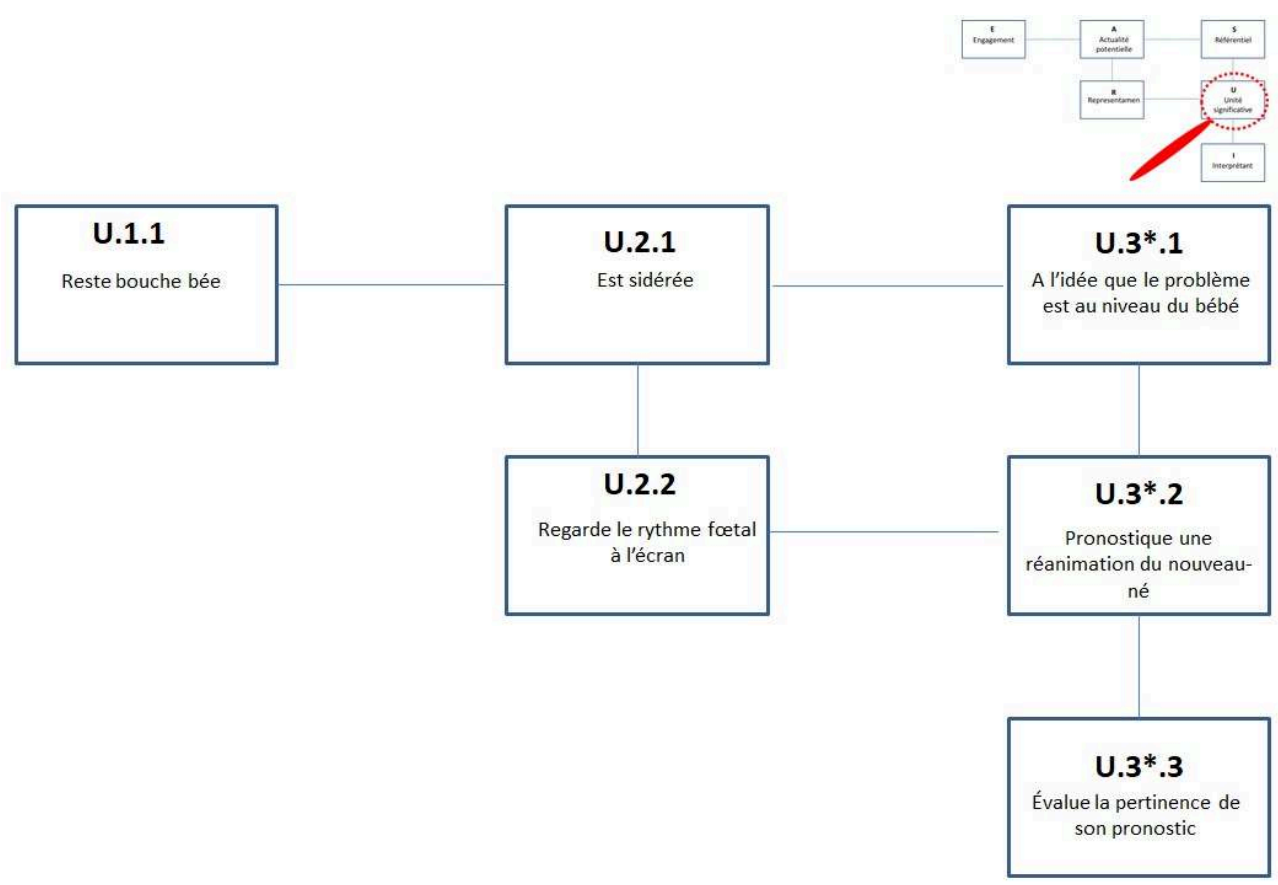

Annexe 2 : Etonnée face à l'aménagement de la table de réanimation.

Appendix 2: Astonished in the face of the resuscitation table setting

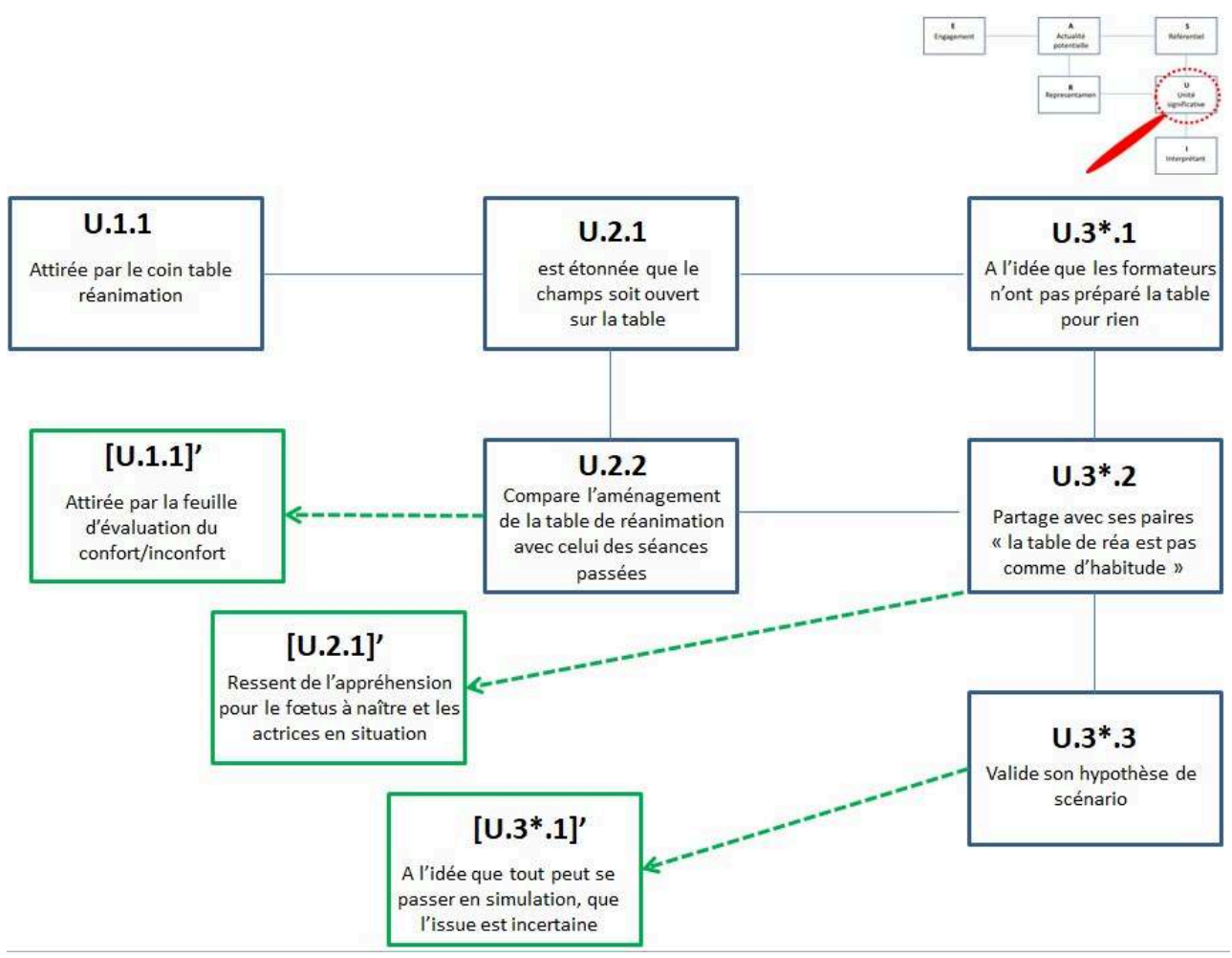


Annexe 3 : Ressent la situation d'inconfort du fœtus et de la SF. Appendix 3: Experiences the discomfort situation of the foetus and the midwife

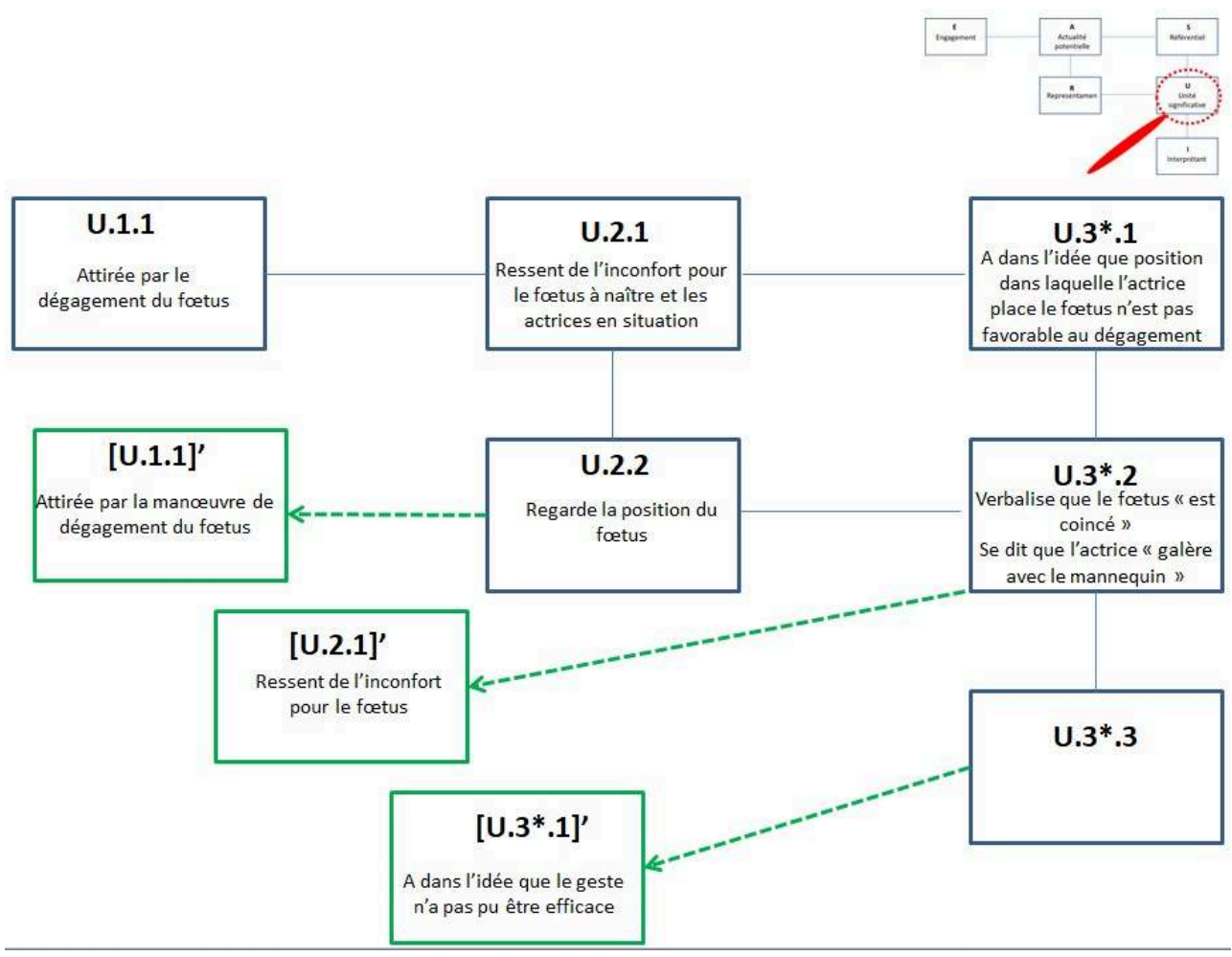

Annexe 4 : Réalise la manœuvre de restitution

Appendix 4: Performs the Couder manoeuvre

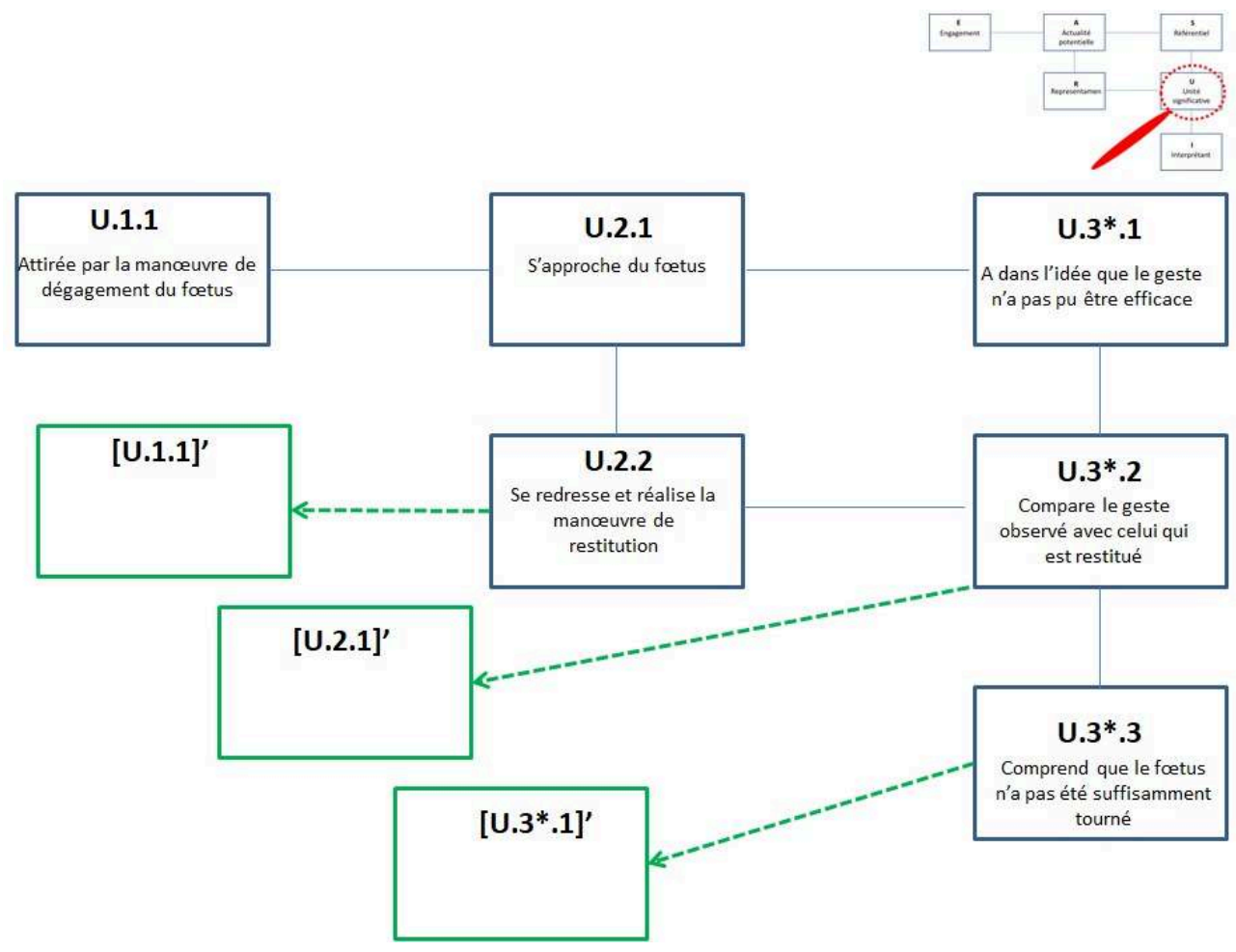


Annexe 5 : Hexatomie du U d'un signe Recherche/Histoire/Passion.

Annexe 5: $U$ hexatomy of a Research/Story/Passion sign

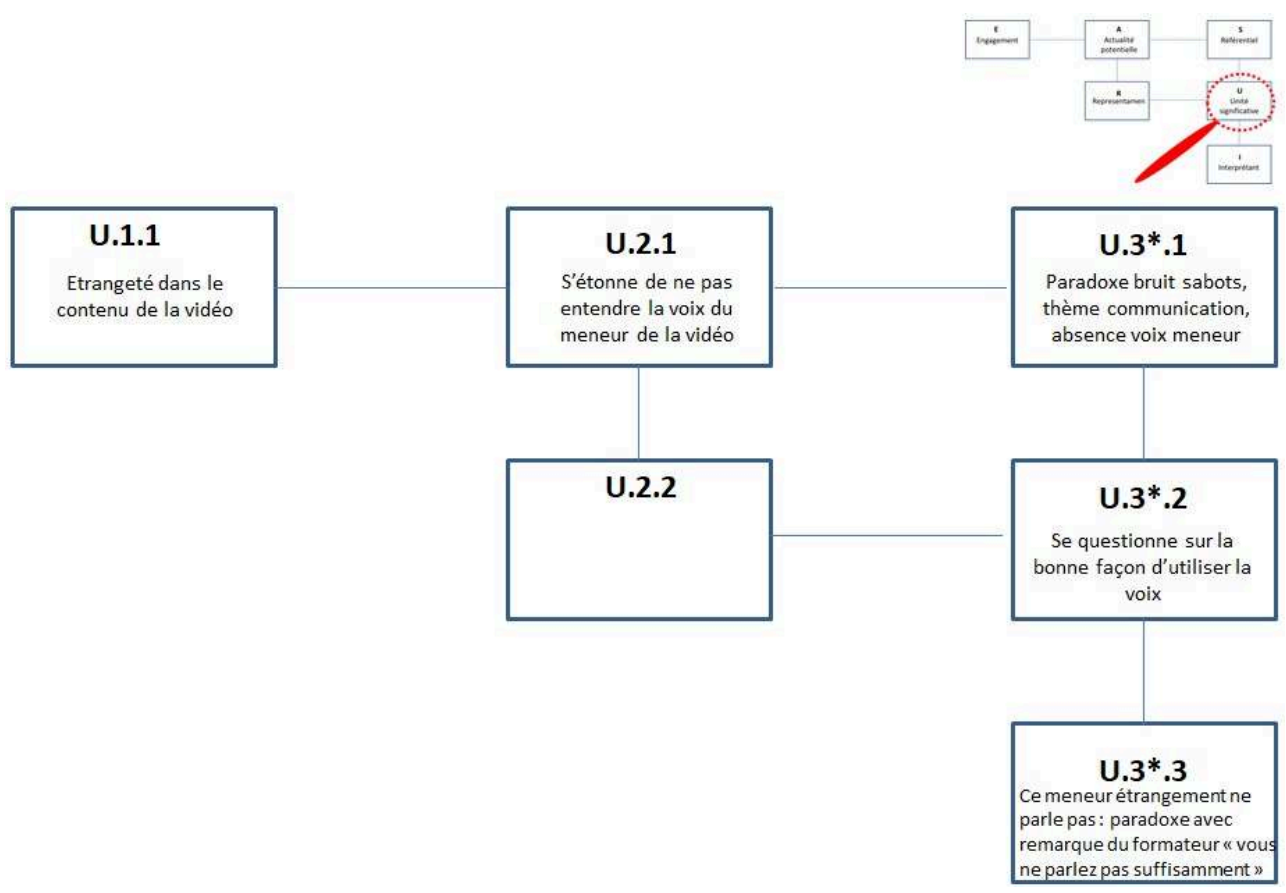

\section{NOTES}

1. L'alloconfrontation réfère ici à une modalité didactico-pédagogique consistant à placer un apprenant en situation d'observer l'activité d'un pair, afin de potentialiser des apprentissages. Il ne s'agit pas d'un dispositif méthodologique mobilisable dans une visée de recherche (Mollo, 2004).

2. Selon le programme de recherche cours d'action, l'activité, est définie comme cognitive, autonome, incarnée, située dynamiquement dans un monde où existent d'autres acteurs, techniquement constituée, cultivée et vécue (Theureau, 2006, p. 40).

3. Les types sont le résultat d'un processus de typicalisation à travers lequel une personne reconnait certaines expériences singulières comme récurrentes dans des contextes perçus comme similaires. Ils se décrivent comme des «schèmes typiques d'attention, de perception, d'action, de communication, d'interprétation et d'émotions " (Theureau, 2000, p. 185) toujours liés à l'activité en cours.

4. Cette double dynamique fonctionne dans les deux sens : certaines expériences passées sont appelées lors de la situation d'allo-confrontation, des expériences vécues en vidéo-formation ou simulation sont mobilisées en situation de pratique réelle.

5. Plateforme de vidéoformation qui organise des traces d'activité réelle d'enseignants faites d'extraits de films, de photos, d'entretiens pour servir de ressources au développement professionnel (Ria, 2010). http://neo.ens-lyon.fr/neopass/connexion.php

6. La formation étudiée concerne la formation professionnelle des cochers-meneurs dispensée par l'Institut Français du Cheval et de l'Équitation (IFCE). Le certificat de spécialisation option «Utilisation et conduite d'attelages de chevaux » est un titre du ministère chargé de l'agriculture qui atteste d'une qualification professionnelle. Le cocher-meneur emploie des chevaux pour transporter du public ou réaliser des services agricoles ou d'aménagement. La formation prévoit 125 heures de technique attelée mais le nombre d'heures de conduite effective par stagiaire est bien inférieur. 
7. L'environnement de formation a été enrichi de deux nouveaux outils pédagogiques : une plateforme de vidéoformation nommée Ercam et un simulateur de guides interactives nommé CoMtacts.

8. La notion de performativité renvoie selon Wulf $(2007$, p. 93) « à l'aptitude du corps à construire un monde » qui « s'exprime dans la langue et dans l'agir social ».

9. «Nous présentons ici l'ordre de construction du chercheur qui n'est pas nécessairement l'ordre temporel de la pratique car, en acte, il n'y a pas d'ordre temporel, le signe fait apparaitre ces composantes dans un processus global continu » (Theureau, 2006, p. 289).

10. Deux thèses récentes, s'inscrivant dans le cadre du programme de recherche du cours d'action, ont été les premières à exploiter l'utilisation des hexatomies de R, U et I pour traiter un corpus de données en lien avec un objet d'étude particulier: l'activité improvisationnelle d'enseignants débutants (Azéma, 2015) et la culture d'action des enseignants (San Martin, 2015).

11. La relation avec un nouvel élément du signe transforme les éléments précédents. Exemple : la relation E-R transforme E en E' (Theureau, 2006, p. 282).

12. Relation ordonnée entre processus: le processus aval dépend pour son existence du processus amont (ce qui signifie, par exemple, que si j'observe un [U.2.2 c'est que les [U.1.1] [U. 2.1] [U.3*.1] l'ont succédé). Relation « sur fond de » $\rightarrow$ (Theureau, 2006, p. 282).

13. Les gestes « iconiques » reproduisent au moins partiellement les gestes actuels ou la forme ou le mouvement d'un objet, ou indiquent sa localisation spatiale (par exemple le mime du mouvement de frapper un obstacle alors que l'acteur relate l'expérience d'un accident) (Petitmengin, 2006, p. 247).

14. «Nommé action symbolique (c'est-à-dire accompagnée de discours privé, et qui peut se réduire à une imagination accompagnée de discours privé ou même à un discours privé tout court, qui constituent des actions de l'acteur sur lui-même) ou communication » (Theureau, 2006, p. 286).

15. Selon Henriot (1983), la conduite ludique a pour caractère le plus apparent, de s'accompagner d'une marge d'imprévisibilité : jouer c'est ne pas savoir où l'on va. L'attitude ludique, c'est aussi faire semblant de croire que la situation simulée est réelle (illusion) et de se détacher du monde dans lequel elle est initialement engagée (duplicité) pour s'immerger et expérimenter une situation diachronique, composé de séquences praxiques plus ou moins aléatoires dont les conséquences seront irréversibles (Ibid.).

16. Ces résultats font écho à ceux produits dans une recherche qui étudie un dialogue d'autoconfrontation croisée entre ouvriers dans le secteur du bâtiment (Duboscq, \& Clot, 2010). Dans l'autoconfrontation croisée, les ouvriers réalisent des nouveaux gestes « à blanc ». Pour les chercheurs, ces gestes, après avoir été utilisés comme «instruments d'argumentation et d'analyse » servent « d'instrument pratique » (Ibid., p. 280).

17. Roche et Gail-Petitfaux (2017) ont étudié différentes situations de visionnage dans un environnement de formation multimodal en EPS comprenant des vidéos prises à partir de différents plans : grand angle, $360^{\circ}$, embarqué et vidéo synchronisée (plan embarqué et plan en grand angle montés dans la même vidéo). Leurs résultats confirment que le visionnement de vidéo de type embarquée est source d'une expérience immersive dans la classe (Sherin, Russ, Sherin, \& Colestock, 2008).

18. Le terme dégénéré, dans le cas de l'unité d'expérience qui est de la catégorie de l'Actuel, signifie qu'il lui est associé une caractéristique qui la renvoie à une catégorie du Potentiel. On a donc un Actuel Potentiel ou un Actuel aux contours faiblement déterminés.

19. Le développement des compétences serait lié à une permanence de l'engagement des acteurs qui enquêtent et cherchent à établir des relations entre différentes expériences au sein d'histoires (Terré, Sève, Saury, 2016). «C'est en s'engageant dans des histoires que les actions et interprétations des élèves prennent une épaisseur temporelle et qu'ils construisent des 
« dispositions à agir » (Durand, 2013) constitutives de compétences » (Terré, Sève, \& Saury, 2016, p. 81).

20. Une étude réalisée dans le cadre de la clinique de l'activité a montré que la répétition des situations intersubjectives d'observation associées à la méthode d'autoconfrontation croisée permettait de « construire de l'objectivité en redéfinissant entre les professionnels un périmètre dialogique qu'ils ne soupçonnaient pas » (Bonnemain, Perrot, \& Kostulski, 2015, p. 121).

\section{RÉSUMÉS}

Cet article présente une réflexion théorique pour étudier les processus d'apprentissagedéveloppement à l'œuvre dans des environnements de formation qui confrontent intentionnellement les formés au monde d'autrui. Ces environnements conçus à partir de films du travail réel ou à partir d'observation en situation de simulation et/ou de pratiques accompagnées sollicitent l'articulation de processus mimétiques, réflexifs, projectifs et ludiques. Au cours de la période 2011-2020, trois études relatives à des situations de vidéoformation et/ou de simulation dans des domaines professionnels différents (formation d'enseignants, de sagesfemmes, de meneurs d'attelage de chevaux) ont été conduites dans le cadre du programme de recherche empirique dit du "Cours d'action" (Theureau, 2004, 2006) ou d'" anthropologie culturelle enactive» (Theureau, 2015). Une modélisation théorique a été développée pour rendre compte de ces processus de mimésis sociale silencieux et complexes (Wulf, 2007) de mises en relation d'expériences vécues par les acteurs (immersion, imitation, émulation, faire le semblable de, représentation, anticipation). Cette réduction théorique basée sur l'hexatomie de l'unité d'action ouvre des pistes méthodologiques à la fois pour mieux saisir les dimensions implicites de l'activité corporelle et sensible très présentes dans ce rapport au monde d'autrui et, en même temps, permet de mieux comprendre les conditions de performativité de l'action (mimésis comme reprise créative vs mimétisme comme copie du réel).

This article presents a theoretical reflection on the study of processes of learning and development at work in the training environments which intentionally cause trainees to consider other peoples' worlds. These environments are based on videos of real work or on the in-situ observation of simulations and/or supported accompanied practices. They solicit articulation between mimetic, reflexive, projective and play processes. Over the 2011-2020 period, three studies on video-training and/or simulation situations in various professional fields (teachers, midwifes and carriage drivers training) were carried out within the framework of the empirical research program known as the "Course of action" (Theureau, 2004, 2006) or as "enactive cultural anthropology" (Theureau, 2015). Theoretical modelling was developed to account for these silent and complex social mimesis processes (Wulf, 2007) of linking agents' experiences (immersion, imitation, emulation, "acting similarly to", representation, anticipation). This theoretical reduction based on the hexatomy of the course of action unit opens up some methodological paths to both better comprehend the implicit dimensions of physical and sensitive activity, that are very highly active in this relation to the world of others and to better understand the conditions of action performativity (mimesis as a creative remake vs mimesis as a copy of reality). 


\section{INDEX}

Mots-clés : expérience mimétique, vidéo-formation, simulation haute-fidélité, alloconfrontation, cours d'expérience

Keywords : mimetic experience, video-training, high fidelity simulation, allo-confrontation, course of experience

\section{AUTEURS}

\section{SERGE LEBLANC}

LIRDEF, Université Montpellier et Université Paul Valéry, Faculté d'éducation, Montpellier. ESPE, de l'académie de Montpellier. FDE. 2 place Marcel Godechot. BP 4152. 34092 Montpellier Cedex 5. serge.leblanc@umontpellier.fr

\section{HÉLÈNE BOUCHOT}

LIRDEF, Département de Maïeutique Site de Nîmes, UFR de Médecine, Université Montpellier. 186 chemin du Carreau de Lanes. CS 83021 - 30908 Nîmes Cedex 2.

helene.bouchot@umontpellier.fr

\section{MÉLANIE SECHEPPET}

LIRDEF, Université Montpellier et Université Paul Valéry, Faculté d'éducation, Montpellier. ESPE, de l'académie de Montpellier. FDE. 2 place Marcel Godechot. BP 4152. 34092 Montpellier Cedex 5. melanie.secheppet@umontpellier.fr 